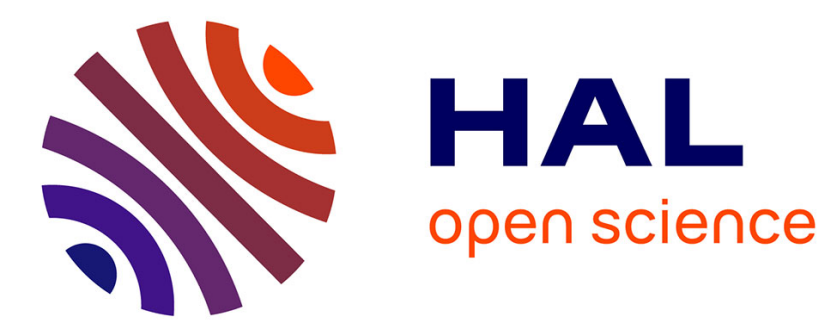

\title{
Computing estimates on material properties from transmission eigenvalues
}

Giovanni Giorgi, Houssem Haddar

\section{To cite this version:}

Giovanni Giorgi, Houssem Haddar. Computing estimates on material properties from transmission eigenvalues. [Research Report] RR-7729, INRIA. 2011. inria-00619232

\section{HAL Id: inria-00619232 \\ https://hal.inria.fr/inria-00619232}

Submitted on 5 Sep 2011

HAL is a multi-disciplinary open access archive for the deposit and dissemination of scientific research documents, whether they are published or not. The documents may come from teaching and research institutions in France or abroad, or from public or private research centers.
L'archive ouverte pluridisciplinaire HAL, est destinée au dépôt et à la diffusion de documents scientifiques de niveau recherche, publiés ou non, émanant des établissements d'enseignement et de recherche français ou étrangers, des laboratoires publics ou privés. 


\title{
I N R I A
}

INSTITUT NATIONAL DE RECHERCHE EN INFORMATIQUE ET EN AUTOMATIQUE

\section{Computing estimates on material properties from transmission eigenvalues}

\author{
Giovanni Giorgi — Houssem Haddar
}

$\mathbf{N}^{\circ} \mathbf{7 7 2 9}$

April 2011

Thème NUM 



\title{
Computing estimates on material properties from transmission eigenvalues
}

\author{
Giovanni Giorgi*, Houssem Haddar ${ }^{\dagger}$ \\ Thème NUM — Systèmes numériques \\ Équipes-Projets DeFI \\ Rapport de recherche $\mathrm{n}^{\circ} 7729$ - April 2011 - 28 pages
}

\begin{abstract}
This work is motivated by inverse scattering problems, those problems where one is interested in reconstructing the shape and the material properties of an inclusion from electromagnetic farfields measurements. More precisely we are interested in complementing the so called sampling methods, (those methods that enables one to reconstruct just the geometry of the scatterer), by providing estimates on the material properties. We shall use for that purpose the so-called transmission eigenvalues. Our method is based on reformulating the so-called interior transmission eigenvalue problem into an eigenvalue problem for the material coefficients. We shall restrict ourselves to the two dimensional setting of the problem and treat the cases of both TE and TM polarizations. We present a number of numerical experiments that validate our methodology for homogeneous and inhomogeneous inclusions and backgrounds. We also treat the case of a background with absorption and the case of scatterers with multiple connected components of different refractive indexes.
\end{abstract}

Key-words: direct and inverse scattering problem, linear sampling method, transmission eigenvalues

* Universita' degli Studi di Genova

† INRIA Saclay Ile de France and Ecole Polytechnique (CMAP)

Centre de recherche INRIA Saclay - Île-de-France

Parc Orsay Université

4, rue Jacques Monod, 91893 ORSAY Cedex

Téléphone : +33172925900 


\section{Rapport de Recherche Inria}

Résumé : Ce travail est motivé par les problèmes de diffraction inverses en lectromagnétisme o l'on est intéressé par la reconstruction de la forme et les propriétés physiques d'une l'inclusion a partir de mesures de champs lointains. Plus précisément, nous nous intéressons à complémenter les méthodes d'échantillonnage (permettant la reconstruction de la géométrie de l'inclusion), en fournissant des estimations sur les propriétés physiques du matériau. Nous utiliserons à cette fin les fréquences de transmission qui sont les valeurs propres du problème de transmission intérieur. Notre méthode est basée sur la reformulation de ce problème en un problème de valeur propre pour l'indice de réfraction. Nous nous limitons dans ce travail au cas bidimensionnel et traitons les deux cas de polarisations TE et TM. Nous présentons un certain nombre d'expériences numériques qui valident notre méthodologie pour les inclusions homogènes et inhomogènes. Nous traitons également le cas d'un milieu de référence avec ou sans absorption ainsi que le cas d'inclusions avec un ou plusieurs composantes connexes possédant des indices de réfraction différents.

Mots-clés : problème de diffraction direct et inverse, linear sampling method, transmission eigenvalues 


\section{Introduction}

This work is motivated by inverse scattering problems, those problems where one is interested in reconstructing the shape and the material properties of an inclusion from electromagnetic farfields measurements. More precisely we are interested in complementing the so called sampling methods [2, 14, (those methods that enables one to reconstruct just the geometry of the scatterer), by providing estimates on the material properties. We shall use for that purpose the so-called transmission eigenvalues [8]. These special frequencies can be determined from the knowledge of the farfields for a range of frequencies. They also correspond to values of the wavenumbers for which the so called homogeneous interior transmission problem has a non trivial solution. Our work is in the same spirit of [11] but we shall employ a different and (in principle) a simpler method.

The proposed method is based on reformulating the interior transmission eigenvalue problem into a (standard, generalized) eigenvalue problem for the material coefficients. In the case of constant coefficients the value of the material parameter corresponds with the smallest eigenvalue [6]. The obtained eigenvalue problem corresponds to a fourth order operator that we shall solve using an appropriate mixed second order formulation of the problem. To obtain the desired approximation of the refractive index, one needs to determine the first transmission eigenvalue from the farfield data. We shall review here the method proposed in 3 , 4 based on a frequency sampling of the farfield solution. We then extend this method to the case of scatterers with multiply connected components so that one is able to detect the transmission eigenvalues associated with each connected components.

In these first investigations we restrict ourselves to a two dimensional setting of the problem that models electromagnetic scattering from infinite cylinders and treat both transverse electric (TE) and transverse magnetic (TM) polarizations. We present a number of numerical experiments that validate our methodology for homogeneous and non homogeneous inclusions and backgrounds. We also treat the case of a background with absorption and the case where the geometry is also unknown (but reconstructed using the linear sampling algorithm).

The outline of this report is the following. The second section is dedicated to introducing the forward and the inverse scattering problem for TM polarizations. We introduce our method for constructing constant approximation of the refractive index in the third section. The fourth section corresponds with the extension of our results to the case of TE polarizations. The last section is dedicated to numerical experiments that validate our approach and illustrate its effectiveness. We finally provide in the appendix a fast implementation procedure of the linear sampling method in the case of backgrounds with piecewise constant and axisymmetric refractive indexes. The latter has been used for instance to perform some of the numerical experimentations in the last section of this report.

\section{Forward and inverse scattering problem}

We consider the scattering of a time harmonic electromagnetic plane wave by an inhomogeneous infinite cylinder with cross section $D$ such that the electric field $E=\left(0,0, u \exp ^{-i \omega t}\right)$ is polarized parallel to the axis of the cylinder (TM polarization). Supposing the index of refraction $n$ dependent only on the two coordinates orthogonal to the cylinder axis and factoring out the time harmonic component we obtain that the total field $u$ satisfies [8]:

$$
\begin{gathered}
\Delta u(x)+k^{2} n(x) u(x)=0 \quad \text { in } \mathbb{R}^{2} \\
u(x)=u^{i}(x)+u^{s}(x), \quad \text { with } u^{i}(x)=\exp ^{i k x \cdot d}, \\
\lim _{r \rightarrow \infty} \sqrt{r}\left(\frac{\partial u^{s}(x)}{\partial r}-i k u^{s}(x)\right)=0 \text { uniformly with respect to } \hat{x}:=\frac{x}{|x|},
\end{gathered}
$$


where $x \in \mathbb{R}^{2}, k>0$ is the wave number, $r=|x|$ and $d \in \Omega=\left\{\frac{x}{|x|}: x \in \mathbb{R}^{2}\right\}$.

The index of refraction $n$ is assumed to be a piecewise continuously differentiable function with discontinuities across non intersecting $\mathcal{C}^{2}$ curves. Moreover we suppose that $\operatorname{Im}(n) \geq 0$ and that, with $m:=1-n$ and $\bar{D}$ being the support of $m, \bar{D}$ is compact with the complement of $D$ connected and boundary $\partial D$ smooth.

Problem (1)-(3) has a unique solution $u \in H_{l o c}^{2}\left(\mathbb{R}^{2}\right)$ and the corresponding scattered field $u^{s}$ has the asymptotic behavior 8 :

$$
u^{s}(x)=\frac{\exp ^{i k r}}{\sqrt{r}} u_{\infty}(\hat{x}, d)+O\left(r^{-3 / 2}\right)
$$

as $r \rightarrow \infty$ uniformly with respect to $\hat{x} \in \Omega$.

The knowledge of the so-called farfield pattern $u_{\infty}(\hat{x}, d)$ for all observation directions $\hat{x} \in \Omega$, all incident directions $d \in \Omega$ and for a range of frequencies $k$ (that will be specified later) forms the data of the inverse problem of determining $D$ and $n$. In fact, although the shape of $D$ will be determined at a fixed frequency $k$, for the reconstruction of $n$, the knowledge of $u_{\infty}(\hat{x}, d)$ for a range of $k$ will be required. As it will be better specified in the following, even in this last case the method will operate without combining data of different frequencies; for this reason we suppressed the mention to $k$ in the notation.

Let $\Phi$ be the radiating fundamental solution of the Helmholtz equation

$$
\Phi(x, y)=\frac{i}{4} H_{0}^{(1)}(k|x-y|),
$$

where $H_{0}^{(1)}$ is the Hankel function of the first kind of order 0 and consider

$$
\Phi_{\infty}(\hat{x}, z):=\frac{\exp ^{i \pi / 4}}{\sqrt{8 \pi k}} \exp ^{-i k \hat{x} \cdot z}
$$

the farfield pattern of $\Phi(\cdot, z)$. Central to the Linear Sampling Method is the farfield equation

$$
\left(F g_{z}\right)(\hat{x})=\Phi_{\infty}(\hat{x}, z)
$$

where the farfield operator $F: L^{2}(\Omega) \rightarrow L^{2}(\Omega)$ is defined by:

$$
(F g)(\hat{x})=\int_{\Omega} u_{\infty}(\hat{x}, d) g(d) d s(d)
$$

We recall that $F g$ is the farfield associated to the incident wave

$$
v_{g}(x):=\int_{\Omega} \exp ^{i k x \cdot d} g(d) d s(d)
$$

called Herglotz wave function with density $g$. We recall hereafter the main theorem associated with the LSM (see [7] for instance). We shall assume in the sequel that $\operatorname{Re}(n)-1$ or $1-\operatorname{Re}(n)$ is positive definite on $D$.

Theorem 2.1. Except possibly for a discrete set of values of $k$ when $\operatorname{Im}(n)=0$, it holds:

1. if $z \in D$, for every $\epsilon>0$ there is a solution $g_{z}^{\epsilon} \in L^{2}(\Omega)$ of the inequality

$$
\left\|F g_{z}^{\epsilon}-\Phi_{\infty}(\cdot, z)\right\|_{L^{2}(\Omega)}<\epsilon
$$

such that

$$
\lim _{\epsilon \rightarrow 0}\left\|v_{g_{z}^{\epsilon}}\right\|_{L^{2}(D)}<\infty
$$

and that

$$
\lim _{z \rightarrow \partial D}\left\|v_{g_{z}^{\epsilon}}\right\|_{L^{2}(D)}=\infty .
$$


2. if $z$ is not in $D$, then for every $\epsilon>0$ and $g_{z}^{\epsilon}$ satisfying

$$
\left\|F g_{z}^{\epsilon}-\Phi_{\infty}(\cdot, z)\right\|_{L^{2}(\Omega)}<\epsilon
$$

we have that

$$
\lim _{\epsilon \rightarrow 0}\left\|v_{g_{z}^{\epsilon}}\right\|_{L^{2}(D)}=\infty
$$

The linear sampling method consists in solving the regularized ( $F$ is compact between infinite dimensional spaces) farfield equation for each $z$ in a grid containing $D$ and visualizing the indicator function $z \mapsto\left\|g_{z}\right\|_{L^{2}(\Omega)}$. This method therefore solves the inverse scattering problem of determining the shape of an unknown object $D$ from the knowledge of its farfield pattern $u_{\infty}$ for all observation directions and all incident directions at a given frequency. $n$. However its fails to do so if this frequency coincides with so called transmission eigenvalues that will be defined in the sequel.

We recall that for $z \in \mathbb{R}^{2}$, the equation $F g_{z}=\Phi_{\infty}(\cdot, z)$ has a solution $g_{z} \in L^{2}(\Omega)$ if and only if there exists $w \in H^{2}(D)$ and a Herglotz wave function $v_{g_{z}}$ such that $w$ and $v_{g_{z}}$ are solution of

$$
\begin{gathered}
\Delta w+k^{2} n w=0, \quad \Delta v_{g_{z}}+k^{2} v_{g_{z}}=0 \quad \text { in } D, \\
w-v_{g_{z}}=\Phi(\cdot, z), \quad \frac{\partial w}{\partial \nu}-\frac{\partial v_{g_{z}}}{\partial \nu}=\frac{\partial \Phi}{\partial \nu}(\cdot, z) \quad \text { on } \partial D .
\end{gathered}
$$

Equations 15-16 form the so called interior transmission problem. As it will be better clarified in the next section, transmission eigenvalues identify wave numbers for which the farfield operator may not be complete in $L^{2}(\Omega)$. In particular, when $k$ is such that the homogeneous problem associated to equations (15)- 16 admits a non trivial solution $(w, v)$ with $v$ a Herglotz wave function, then farfield operator $F$ is not complete in $L^{2}(\Omega)$ [8]. In [6] the set of transmission eigenvalues has been proved to be infinite and countable.

The case of inhomogeneous background. We conclude this section by noting that an adaptation of the linear sampling method to the problem of detecting an object embedded in an inhomogeneous background has been well established for example in 7] (for TM polarized plane waves and impenetrable objects) and in [9] (for near field measurements and penetrable objects). In the inhomogeneous cases, the kernel of the farfield operator has to be modified by subtracting its background analogous and the right hand side should be replaced by the farfield of the background Green's function. The index $n_{b}$ of the inhomogeneous background is assumed to verify similar regularity assumptions as $n$ and to be equal to 1 outside a ball.

Following [7] if $u_{b, \infty}^{s}$ denotes the farfield pattern of the background medium (i.e. without the inclusion $D)$ and if $G_{\infty}(\cdot, z)$ is the farfield of the Green function corresponding to the inhomogeneous background and to a point source at $z$, then Theorem 2.1 holds with

$$
(F g)(\hat{x}):=\int_{\Omega}\left[u_{\infty}(\hat{x}, d)-u_{b, \infty}^{s}(\hat{x}, d)\right] g_{z}(d) d s(d)
$$

and $\Phi_{\infty}$ replaced by $G_{\infty}$. If $u_{b}(\cdot, d)$ denotes the total field associated with the background, i.e. solution to (1)-(3) with $n=n_{b}$, then the Herglotz wave is defined in the present case as

$$
v_{g}(x):=\int_{\Omega} u_{b}(x, d) g(d) d s(d)
$$

and forms the incident wave that generates, for the inclusion $D$, a farfield equals to $F g$. 


\section{Derivation of a constant approximation of the refractive index}

We consider the case of a non-absorbing (i.e. $\operatorname{Im}(n)=0$ ) inhomogeneous medium embedded in a (possibly inhomogeneous) background and we assume that LSM or other qualitative methods 2, 14. have already been used to determine the support $D$ of the target. The aim of this section is to determine an estimate of the index of refraction $n(x)$ under the assumption that the support $D$ is known.

To achieve this goal we shall make use of the first transmission eigenvalue and consider the interior transmission eigenvalue problem as an eigenvalue problem where the eigenvalue is the index of refraction. This is why we first need to provide a clearer definition of transmission eigenvalues and comment on their identification using farfield data.

\subsection{Transmission eigenvalues and their identification using farfield data}

We start analyzing the case of a TM polarized scattering problem of an inhomogeneous medium in the vacuum. Using the notation and assumptions of the second section, we define the interior transmission problem [8, 10] as the problem of finding $w, v \in L^{2}(D)$, with $w-v \in H^{2}(D)$, such that

$$
\begin{array}{rr}
\Delta w+k^{2} n w=0 & \text { in } D \\
\Delta v+k^{2} v=0 & \text { in } D \\
w-v=f & \text { on } \partial D \\
\frac{\partial w}{\partial \nu}-\frac{\partial v}{\partial \nu}=g & \text { on } \partial D
\end{array}
$$

for some boundary data $f \in H^{\frac{1}{2}}(\partial D)$ and $g \in H^{\frac{3}{2}}(\partial D)$.

We have that the farfield operator $F$ is injective with dense range provided that $k$ is not a transmission eigenvalue, i.e. a value of $k$ for which the homogeneous (i.e. $f=0$ and $g=0$ in (21) and 221) interior transmission problem has a non trivial solution. We also recall that for $z \in D$, the farfield equation has a nearby solution $g_{z}^{\epsilon} \in L^{2}(\Omega)$ that satisfies $(10)-(11)$ if there exists a couple $\left(w_{z}, v_{z}\right)$ solution of 19$)-(22)$ with $f=\Phi(\cdot, z)$ and $g=\frac{\partial}{\partial \nu} \Phi(\cdot, z)$.

Since the linear sampling method is expected to fail when $k$ is a transmission eigenvalue and, in particular, for almost all $z \in D$ the norm of a regularized solution $g_{z, \alpha}$ satisfying

$$
\lim _{\alpha \rightarrow 0}\left\|F g_{z, \alpha}-\Phi_{\infty}(\cdot, z)\right\|_{L^{2}(\Omega)}=0
$$

is expected to be large for such values of $k$ [5, 3], a possible method for finding transmission eigenvalues would be to solve (23) for few fixed $z \in D$ and for values of $k$ in an interval: the transmission eigenvalues would correspond with large values of $k \mapsto\left\|g_{z}\right\|_{L^{2}(\Omega)}$.

The following theorem formalizes previous considerations (the formulation is an adaptation of the results in [5]).

Theorem 3.1. Let $g_{z, \alpha}$ satisfy (23) and $z$ be a point of $\mathbb{R}^{2}$; let $v_{g_{z, \alpha}}$ be the Herglotz wave function associated to $g_{z, \alpha}$ and let us assume that $k$ is a transmission eigenvalue. Then for almost every $z \in D,\left\|v_{g_{z, \alpha}}\right\|_{L^{2}(D)}$ cannot be bounded as $\alpha \rightarrow 0$. Otherwise, if $k$ is not a transmission eigenvalue, then there exists $g_{z, \alpha}$ satisfying (23) such that $\left\|v_{g_{z, \alpha}}\right\|_{L^{2}(D)}$ is bounded as $\alpha \rightarrow 0$ for each $z \in D$.

Let us denote by $P(D, k, n)$ the interior transmission problem 19]-22 for a domain $D$ of refractive index $n$ and with boundary data $f=\Phi(\cdot, z)$ and $g=\frac{\partial}{\partial \nu} \Phi(\cdot, z)$.

We give in following a corollary of the previous theorem that indicates how, for multiply connected inclusions $D$, one can identify the transmission eigenvalues associated with each connected component. More precisely, assume that $D=\bigcup_{\hat{j} \in\left\{j_{0}, \ldots, j_{m}\right\}} D_{\hat{j}}$ with $m \in \mathbb{N}$ and $D_{\hat{j}}$ connected for each $\hat{j} \in\left\{j_{0}, \ldots, j_{m}\right\}$ and refer to $P\left(D_{\hat{j}}, k, n_{\hat{j}}\right)$ as the interior transmission problem associated to 
$D_{\hat{j}}$, with $n_{\hat{j}}$ restriction of $n$ to $D_{\hat{j}}$. We remark that, obviously, the set of transmission eigenvalues for $D$ is the union of transmission eigenvalues for $D_{\hat{j}}$ for $\hat{j} \in\left\{j_{0}, \ldots, j_{m}\right\}$.

Let us consider the farfield equation associated to the whole $D$ and let $g_{z, \alpha}$ satisfy [23].

Corollary 3.1. Let $j_{p}, j_{q} \in\left\{j_{0}, \ldots, j_{m}\right\}$ and let $k^{*}$ be a transmission eigenvalue for $P\left(D_{j_{p}}, k, n_{j_{p}}\right)$ and not for $P\left(D_{j_{q}}, k, n_{j_{q}}\right)$. Then

- $\left\|v_{g_{z, \alpha}}\right\|_{L^{2}(D)}$ is unbounded for almost every $z \in D_{j_{p}}$.

- For each $z \in D_{j_{q}}$ there exists a $g_{z, \alpha}$ satisfying 23) such that $\left\|v_{g_{z, \alpha}}\right\|_{L^{2}(D)}$ is bounded.

Proof. First, we point out that if $(w, v)$ is a solution of $P(D, k, n)$, then $\left(w_{\bar{j}}, v_{\bar{j}}\right)$ (restriction of $(w, v)$ to $\left.D_{\bar{j}}\right)$ would be a solution of $P\left(D_{\bar{j}}, k, n_{\bar{j}}\right)$ and that

$$
\|v\|_{L^{2}(D)}^{2}=\sum_{\bar{j} \in\left\{j_{0}, \ldots, j_{m}\right\}}\left\|v_{\bar{j}}\right\|_{L^{2}\left(D_{\bar{j}}\right)}^{2} .
$$

- Let $k^{*}$ be a transmission eigenvalue for $P_{j_{p}}$. Then, from Theorem 3.1, it follows that $\left\|v_{g_{z, \alpha}, j_{p}}\right\|_{L^{2}\left(D_{j_{p}}\right)}$ has to be unbounded for almost every $z \in D_{j_{p}}$. Then, by (24), $\left\|v_{g_{z, \alpha}}\right\|_{L^{2}(D)}$ is unbounded for almost every $z \in D_{j_{p}}$.

- If $z \in D_{j_{q}},(0, \Phi(\cdot, z))$ is a (bounded) solution of $P_{j_{p}}$. Hence, $(w, v)$ such that $w=0$ on $D$, $v=0$ on $D_{\bar{j}}$ for each $\bar{j} \neq j_{p}$ and $v=\Phi(\cdot, z)$ on $D_{j_{p}}$ is a bounded solution of $P\left(D, k^{*}, n\right)$. Denseness of the set of Herglotz wave functions implies the existence of a $g_{z, \alpha}$ such that $\left\|v_{g_{z, \alpha}}-v\right\|_{L^{2}(D)} \rightarrow 0$ for $\alpha \rightarrow 0$. This implies that $\left\|F g_{z, \alpha}-\Phi(\cdot, z)\right\|_{L^{2}(\Omega)} \rightarrow 0$ for $\alpha \rightarrow 0$.

\subsection{Identification of the refractive index}

As explained before, solving the interior transmission eigenvalue problem means, knowing the shape of $D$ and the pointwise values of $n$, find $k^{*}$ such that there exists a non trivial solution $u$ of the problem $P(D, k, n)$.

Let us change the point of view: since transmission eigenvalues are detectable without knowing $n(x)$, it is possible to consider directly the problem $P\left(D, k^{*}, n\right)$ where $D$ and $k^{*}$ are known and $n$ is unknown. Moreover, supposing $n$ constant, $P\left(D, k^{*}, n\right)$ could be treated as a classical generalized eigenvalue problem.

Now, we are ready to explain the idea of the whole method. Given the inverse scattering problem of detecting an inhomogeneous medium of index $n$, one can first reconstruct the shape of the unknown object $D$ using the LSM (or other similar qualitative methods). Then, fixing a $z$ in $D$ and plotting the norm of $g$ (solution of (23p) for every $k$ in an interval, transmission eigenvalues $k^{*}$ of $P(D, k, n)$ can be detected. With their knowledge, it will be possible to solve the problem of finding $n^{*}$ so that $P\left(D, k^{*}, n^{*}\right)$ has a non trivial solution; i.e. finding $n^{*}$ for which $k^{*}$ is a transmission eigenvalue of $P\left(D, k, n^{*}\right)$.

Proposition 3.1. Assume that $n$ is constant. Then, the homogeneous interior transmission problem $P(D, k, n)$ is equivalent to the problem of finding $u \in H_{0}^{2}(D)$ satisfying

$$
\left(\Delta+k^{2}\right) \Delta u=-k^{2} n\left(\Delta+k^{2}\right) u \quad \text { in } D .
$$

Further, the eigenvalue problem: find $(u, n) \in H_{0}^{2}(D) \times \mathbb{R}$ satisfying 25, admits a countable number of eigenvalues $n \in \mathbb{R}$.

Proof. The equivalence between 200-22) (with $n$ constant) and 25) can be easily proved by setting $u=w-v([2,15])$.

Problem 25) is equivalent to $\left(\Delta+k^{2}\right)^{2} u=\lambda\left(\Delta+k^{2}\right) u$ with $\lambda=k^{2}(1-n)$. The operator $\left(\Delta+k^{2}\right)^{2}: H_{0}^{2}(D) \mapsto H_{0}^{2}(D)$ is bijective and self adjoint while operator $\left(\Delta+k^{2}\right): H_{0}^{2}(D) \mapsto H_{0}^{2}(D)$ is compact, injective and self adjoint. Then, the existence of a countable number of real eigenvalues $\lambda$ can be proved applying classical spectral theory for compact self adjoint operators. 
The main difficulty related to numerical solutions of the eigenvalue problem (25) comes from the fourth order operator which requires the use of $C^{1}$ finite elements. We shall in the following provide mixed formulations of the problem that allows the use of $C^{0}$ finite elements. These formulations, inspired by classical mixed formulation for the biharmonic problem, are those used in the numerical simulations (done with standard $P_{1}$ Lagrange finite elements).

Proposition 3.2. Assume that $n$ is constant. Then if $u \in H_{0}^{2}(D) \cap H^{3}(D)$ is such that (25) is valid, then $u$ and $\mathrm{U}=\left(\Delta+k^{2}\right) u$ are solution of the variational problem: "Find $u \in H_{0}^{1}(D)$ and $\mathrm{U} \in H^{1}(D)$ such that

$$
\int_{D}\left(\mathrm{UV}+\nabla u \nabla \mathrm{V}-k^{2} u \mathrm{~V}-\nabla \mathrm{U} \nabla v\right) d x=-k^{2} n \int_{D}\left(k^{2} u v-\nabla u \nabla v\right) d x
$$

for all $v \in H_{0}^{1}(D), \mathrm{V} \in H^{1}(D) "$.

Conversely, if $u \in H_{0}^{1}(D) \cap H^{3}(D)$ and $\mathrm{U} \in H^{2}(D)$ are solutions of (26), then $u \in H_{0}^{2}(D)$ and satisfies (25).

Proof. Substituting $\mathrm{U}=\left(\Delta+k^{2}\right) u$, equation 25 becomes $\Delta \mathrm{U}=-k^{2} n \mathrm{U}$. Multiplying both equations respectively against test functions $\mathrm{V} \in H^{1}(D)$ and $v \in H_{0}^{1}(D)$ and applying Green's identity we obtain

$$
\int_{D}\left(\nabla u \nabla \mathrm{V}-k^{2} u \mathrm{~V}\right) d x=-\int_{D} \mathrm{UV} d x
$$

and

$$
-\int_{D} \nabla \mathrm{U} \nabla v d x=-k^{2} n \int_{D}\left(k^{2} u v-\nabla u \nabla v\right) d x .
$$

Summing the two equations we obtain $(26)$.

Reciprocally, starting with 26 we can go back to equations 25 by assuming that $u \in H_{0}^{1}(D) \cap$ $H^{3}(D)$ and $\mathrm{U} \in H^{2}(D)$. Using Green's identity we obtain that (27) is equivalent to

$$
\int_{D}\left(\mathrm{U}-\Delta u-k^{2} n u\right) \mathrm{v} d x=\int_{\partial D} \frac{\partial u}{\partial \nu} \mathrm{V} d s(x)
$$

for all $\mathrm{v} \in H^{1}(D)$. Since $\mathcal{C}_{c}^{\infty}(D) \subset H^{1}(D)$ we get $\int_{D}\left(\mathrm{U}-\Delta u-k^{2} n u\right) \mathrm{v} d x=0$ for all $\mathrm{v} \in \mathcal{C}_{c}^{\infty}(D)$. Hence, $\mathrm{U}-\Delta u-k^{2} n u=0$ almost everywhere in $D$ and consequently $\frac{\partial u}{\partial \nu}=0$ almost everywhere on $\partial D$. The latter implies in particular that $u \in H_{0}^{2}(D)$. Similar arguments applies to 28 and prove that $\Delta \mathrm{U}=-k^{2} n \mathrm{U}$ almost everywhere in $D$, which implies 25.

\subsection{The case of an inhomogeneous background}

The approach described in the previous subsection can be extended to the case in which the medium is embedded in an inhomogeneous background. Let us consider the case where the inclusion $D$ is embedded in a background with index $n_{b}$. Then the homogeneous interior transmission problem is formulated as follows:

$$
\begin{array}{rr}
\Delta w+k^{2} n_{D} w=0 & \text { in } D, \\
\Delta v+k^{2} n_{b} v=0 & \text { in } D, \\
w=v & \text { on } \partial D, \\
\frac{\partial w}{\partial \nu}=\frac{\partial v}{\partial \nu} & \text { on } \partial D .
\end{array}
$$

where $n_{D}$ denotes the index of $D$. Taking $u=w-v$ in (30)-33 and applying the operator $\Delta+k^{2} n_{D}$ to the difference $30-31$ we obtain

$$
\left(\Delta+k^{2} n_{D}\right) \frac{1}{k^{2}\left(n_{b}-n_{D}\right)}\left(\Delta+k^{2} n_{b}\right) u=0 \quad \text { in } D
$$




$$
u=0, \quad \frac{\partial u}{\partial \nu}=0 \quad \text { on } \partial D
$$

Substituting

$$
\mathrm{U}:=\frac{1}{k^{2}\left(n_{b}-n_{D}\right)}\left(\Delta+k^{2} n_{b}\right) u
$$

we obtain

$$
\Delta \mathrm{U}=-k^{2} n_{D} \mathrm{U} \quad \text { in } D .
$$

Multiplying (37) by a test function $v \in H_{0}^{1}(D)$ and $(36)$ by $k^{2}\left(n_{b}-n_{D}\right) \mathrm{V}$ with $\mathrm{v} \in H^{1}(D)$, applying the Green's formula, taking into account the boundary conditions satisfied by $u$ and adding the two equations, one ends up with the following variational formulation

$$
\int_{D}\left(k^{2} n_{b}(u \mathrm{~V}-\mathrm{UV})-\nabla u \nabla \mathrm{V}-\nabla \mathrm{U} \nabla v\right) d x=\lambda \int_{D}(\mathrm{U} v+\mathrm{UV}) d x
$$

which is valid for all $v \in H_{0}^{1}(D)$ and $\mathrm{V} \in H^{1}(D)$ and with $\lambda:=-k^{2} n_{D}$. Using this procedure and similar arguments as in the proof of Proposition 3.2 , one can prove the following.

Proposition 3.3. If $n_{D}$ is assumed to be constant and $\exists w, v \in L^{2}(D)$ and $w-v=: u \in H_{0}^{2}(D) \cap$ $H^{3}(D)$ such that (30)-(33) is valid, then $u$ and $\mathrm{U}=\frac{1}{k^{2}\left(n_{b}-n_{D}\right)}\left(\Delta+k^{2} n_{b}\right) u$ are solution of the variational problem: "Find $u \in H_{0}^{1}(D)$ and $\mathrm{U} \in H^{1}(D)$ such that

$$
\int_{D}\left(k^{2} n_{b}(u \mathrm{~V}-\mathrm{UV})-\nabla u \nabla \mathrm{V}-\nabla \mathrm{U} \nabla v\right) d x=\lambda \int_{D}(\mathrm{U} v+\mathrm{UV}) d x
$$

for all $v \in H_{0}^{1}(D), \mathrm{v} \in H^{1}(D) "$.

Conversely, if $u \in H_{0}^{1}(D) \cap H^{3}(D)$ and $\mathrm{U} \in H^{2}(D)$ are solutions of (39), then $u \in H_{0}^{2}(D)$ and satisfies 34.

Remark 3.1. In the case of inhomogeneous background, (7) takes the form of

$$
\left[F g_{z}\right](\cdot)=G_{\infty}(\cdot, z)
$$

where $G_{\infty}$ and $F$ are defined as (17), taking into account the presence of the inhomogeneous background. As in the homogeneous case, $k \mapsto\left\|g_{z}\right\|_{L^{2}(\Omega)}$ is expected to have peaks for $k$ being a transmission eigenvalue for a.e. $z \in D$. This procedure allows us to compute the transmission eigenvalues from farfield data.

\subsubsection{A remark on the case of absorbing background}

It is well known that, when a non-absorbing object of support $D$ is embedded in a background such that $\operatorname{Im}\left(n_{b}\right)>0$, then there are no real transmission eigenvalues associated with (30)-(33) [8]. Since complex transmission eigenvalues are not detectable by the procedure previously indicated in (Remark 3.1), this means that our method providing estimates on the index of refraction is not applicable.

However, in this preliminary remark something is not sufficiently precise: the definition of the background index inside $D$ has no physical meaning since the "physical index" of the medium inside $D$ is $n$. For backgrounds with constant indexes or for layered backgrounds, a "natural extension" of the definition of the background index inside $D$ can be defined. But this is mainly only for a mathematical or numerical convenience.

This simple consideration suggests that there can be some degrees of freedom in the definition of $n_{b}$ inside $D$, which is the part used in the interior transmission problem (30)- 33 . As far as the inverse problem of determining $n$ is concerned, this is feasible since at this stage we assume that $D$ is known. In particular if we choose $n_{b}$ to be a real constant (different from $n_{D}$ ) in $D$, then we are back again to the case where the interior transmission problem has real eigenvalues 
and therefore one would be able to apply the procedure previously explained to get estimates on $n_{D}$. This procedure will be validated by some numerical experiment in the numerical section.

Of course there is an extra price to pay: it is hidden in the step of determining the transmission eigenvalue from farfield data. Indeed when one complexifies the background index, this makes the computation of $u_{b, \infty}^{s}$ in (17) more costly.

We finally observe that this procedure can also be applied in the case without absorption if one would like to use the (simpler) formulation of the interior transmission problem with constant index.

\section{The case of TE polarization}

Our results for TM polarized waves can be extended to the case of transverse electric (TE) polarization, i.e. the case for which the magnetic component of the field (originated by the incidence of a time harmonic plane wave) vibrates parallel to the axis of the infinite cylinder that is assumed to have cross section $D$. The main steps of the inversion algorithm are the same as in the TM case and we shall hereafter only give a sketch of the main differences.

Let us first quickly introduce the direct scattering problem for a scatterer $D$ of refractive index $n$ in the case of TE incident plane waves. The total field $u \in H_{\text {loc }}^{1}\left(\mathbb{R}^{2}\right)$ satisfies

$$
\begin{gathered}
\nabla \cdot\left(\frac{1}{n(x)} \nabla u(x)\right)+k^{2} u(x)=0 \quad \text { in } \mathbb{R}^{2}, \\
u(x)=u^{i}(x)+u^{s}(x) \quad \text { with } \quad u^{i}(x)=\exp ^{i k x \cdot d}, \\
\lim _{r \rightarrow \infty} \sqrt{r}\left(\frac{\partial u^{s}(x)}{\partial r}-i k u^{s}(x)\right)=0 .
\end{gathered}
$$

The associated farfield pattern as well as the farfield operator can be deduced as in the TM case. Theorem 2.1 is also still valid in the present case under the same assumption on the refractive index.

\subsection{Transmission eigenvalues}

In this case, the homogeneous interior transmission problem is defined as follows [4: find $\tilde{w}, v \in$ $L^{2}(D)$ such that $\tilde{w}-v=: u \in H^{1}(D), \nabla \cdot\left(\frac{1}{n} \nabla \tilde{w}-\nabla v\right) \in L^{2}(D)$ and

$$
\begin{array}{rr}
\nabla \cdot\left(\frac{1}{n} \nabla \tilde{w}\right)+k^{2} \tilde{w}=0 & \text { in } D, \\
\Delta v+k^{2} v=0 & \text { in } D, \\
\tilde{w}=v & \text { on } \partial D, \\
\frac{1}{n} \frac{\partial \tilde{w}}{\partial \nu}=\frac{\partial v}{\partial \nu} & \text { on } \partial D .
\end{array}
$$

Since the Linear Sampling Method works independently from wave polarization, results obtained in the case of transverse magnetic waves as well as Theorem 3.1 and Corollary 3.1 could be extended to the case of transverse electric polarization. Hence, again, transmission eigenvalues are detectable from the knowledge of farfield patterns and the resolution of the eigenvalue problem $P\left(D, k_{0}^{*}, n\right)$ can provide us with a constant approximation $n^{*}$ of $n$.

\subsection{Mixed formulations of the refractive index eigenvalues problems}

We shall explain here how problem 44 -47 can be formulated as an eigenvalue problem for a constant $n$, assuming that $k$ and $D$ are known. 
Proposition 4.1. Considering $n$ constant, problem (44)-(47) is equivalent to the problem of finding $u:=\frac{1}{n} \tilde{w}-v \in H^{2}(D)$ such that

$$
\begin{gathered}
\left(\Delta+k^{2}\right) \Delta u=-k^{2} n\left(\Delta+k^{2}\right) u \quad \text { in } D \\
\Delta u=0, \quad \frac{\partial u}{\partial \nu}=0 \quad \text { on } \partial D .
\end{gathered}
$$

Proof. Substituting $w=\frac{1}{n} \tilde{w}$ and using 44 and 45 it is easy to obtain

$$
\begin{array}{rc}
\Delta w+k^{2} n w=0 & \text { in } D, \\
\Delta v+k^{2} v=0 & \text { in } D, \\
\Delta w=-k^{2} \tilde{w}=-k^{2} v=\Delta v & \text { on } \partial D \\
\frac{\partial w}{\partial \nu}=\frac{\partial v}{\partial \nu} & \text { on } \partial D .
\end{array}
$$

The equivalence between (50)- 53 and $448-(49)$ can be shown as is the TM case.

Proposition 4.2. Assuming $n$ constant then: if $u \in H^{2}(D) \cap H^{3}(D)$ is such that (48)-(49) is valid, then $u$ and $\mathrm{U}:=\Delta u$ are solution of the problem: "Find $u \in H^{1}(D)$ and $\mathrm{U} \in H_{0}^{1}(D)$ such that

$$
\int_{D}\left(\mathrm{U} v+\nabla u \nabla \mathrm{v}+k^{2} \mathrm{UV}-\nabla \mathrm{U} \nabla \mathrm{v}\right) d x=-k^{2} n \int_{D}\left(k^{2} u \mathrm{v}-\nabla u \nabla \mathrm{v}\right) d x
$$

for all $v \in H^{1}(D), \mathrm{v} \in H_{0}^{1}(D)$ ”.

Conversely, if $u \in H^{3}(D)$ and $\mathrm{U} \in H_{0}^{1}(D) \cap H^{2}(D)$ are solutions of (54), then (48)-(49) is satisfied by $u$.

Proof. Substituting $\mathrm{U}=\Delta u$, equation 48 becomes $\left(\Delta+k^{2}\right) \mathrm{U}=-k^{2} n\left(\Delta+k^{2}\right) u$. Multiplying both equations respectively against test functions $v \in H^{1}(D)$ and $\mathrm{v} \in H_{0}^{1}(D)$ and applying Green's identity we obtain

$$
\int_{D} \mathrm{U} v d x=-\int_{D} \nabla u \nabla v d x
$$

and

$$
\int_{D}\left(-\nabla \mathrm{U} \nabla \mathrm{v}+k^{2} \mathrm{UV}\right) d x=-k^{2} n \int_{D}\left(k^{2} u \mathrm{~V}-\nabla u \nabla \mathrm{v}\right) d x .
$$

Summing the two equations we obtain (54). The reverse implication is a classical exercise similar to the one done at the end of the proof of Proposition 3.2 .

\subsubsection{The case of an inhomogeneous background}

The case of a scatterer embedded in an inhomogeneous background illuminated by TE polarized plane waves is more complex. Nevertheless, following the approach of [4], it is possible to write the homogeneous interior transmission problem as a generalized eigenvalue problem with $\lambda=-k^{2} n_{D}$ as eigenvalues.

Let us again consider an inclusion $D$ with index $n_{D}$ embedded in a background with index $n_{b}$; the homogeneous interior transmission problem is formulated as follows:

$$
\begin{array}{rr}
\nabla \cdot\left(\frac{1}{n_{D}} \nabla w\right)+k^{2} w=0 & \text { in } D, \\
\nabla \cdot\left(\frac{1}{n_{b}} \nabla v\right)+k^{2} v=0 & \text { in } D, \\
w=v & \text { on } \partial D, \\
\frac{1}{n_{D}} \frac{\partial}{\partial \nu} w=\frac{1}{n_{b}} \frac{\partial}{\partial \nu} v & \text { on } \partial D .
\end{array}
$$


Taking the gradient of 57 and 587 and substituting $\mathbf{w}=\frac{1}{n_{D}} \nabla w$ and $\mathbf{v}=\frac{1}{n_{b}} \nabla v$ in 57 - 60 . we obtain

$$
\begin{aligned}
\nabla(\nabla \cdot \mathbf{w})+k^{2} n_{D} \mathbf{w}=0 & \text { in } D \\
\nabla(\nabla \cdot \mathbf{v})+k^{2} n_{b} \mathbf{v}=0 & \text { in } D
\end{aligned}
$$

with boundary conditions

$$
\nu \cdot \mathbf{w}=\nu \cdot \mathbf{w} \quad \text { and } \nabla \cdot \mathbf{w}=\nabla \cdot \mathbf{v} \quad \text { on } \partial D .
$$

Setting $\mathbf{u}=\mathbf{w}-\mathbf{v}$ and taking the difference between 61) and 62 we obtain

$$
\frac{1}{k^{2}\left(n_{b}-n_{D}\right)}\left(\nabla \nabla \cdot+k^{2} n_{b}\right) \mathbf{u}=\mathbf{w} .
$$

Then, applying $\left(\nabla \nabla \cdot+k^{2} n_{D}\right)$ to both sides,

$$
\left(\nabla \nabla \cdot+k^{2} n_{D}\right) \frac{1}{k^{2}\left(n_{b}-n_{D}\right)}\left(\nabla \nabla \cdot+k^{2} n_{b}\right) \mathbf{u}=0 \quad \text { in } D .
$$

Substituting $\mathbf{U}=\frac{1}{k^{2}\left(n_{b}-n_{D}\right)}\left(\nabla \nabla \cdot+k^{2} n_{b}\right) \mathbf{u}$ we obtain $\left(\nabla \nabla \cdot+k^{2} n_{D}\right) \mathbf{U}=0$ in $D$, which gives rise to the system

$$
\begin{array}{rr}
\left(\nabla \nabla \cdot+k^{2} n_{b}\right) \mathbf{u}-k^{2} n_{b} \mathbf{U}=-k^{2} n_{D} \mathbf{U} & \text { in } D, \\
\nabla \nabla \cdot \mathbf{U}=-k^{2} n_{D} \mathbf{U} & \text { in } D, \\
\nu \cdot \mathbf{u}=0 \quad \text { and } \quad \nabla \cdot \mathbf{u}=0 & \text { on } \partial D .
\end{array}
$$

The latter eigenvalue problem for eigenvalues $\lambda=-k^{2} n_{D}$ can be formulated in a variational form as in the inhomogeneous TM case by respectively replacing the variational spaces $H^{1}(D)$ and $H_{0}^{1}(D)$ with

$$
H_{\text {div }}(D):=\left\{\mathbf{V} \in L^{2}(D)^{2} ; \nabla \cdot \mathbf{V} \in L^{2}(D)\right\} \ni \mathbf{U},
$$

and

$$
\left\{\mathbf{v} \in H_{\text {div }}(D) ; \nu \cdot \mathbf{u}=0 \text { on } \partial D\right\} \ni \mathbf{u} .
$$

The numerical implementation of the resulting variational formulation requires the use of $H_{\text {div }}$ elements. We did not validate our procedure in the present case.

\section{$5 \quad$ Numerical Experiments}

\subsection{The case where the shape is known}

Before presenting some numerical results concerning the reconstruction of the shape and the constant approximation of the refractive index of unknown objects, let us show how - solving $P\left(D, k_{0}^{*}, n\right)$ as a generalized eigenvalue problem - we obtain a good approximation of $n$ when both $D$ and its first transmission eigenvalue are known.

We focus on the case of a circular geometry and we shall investigate two configurations: in the first one, the refractive index is constant (Figure 1 (a)) and in the second one the index of refraction has two different values in two concentric circles (Figure 1 (b)). 


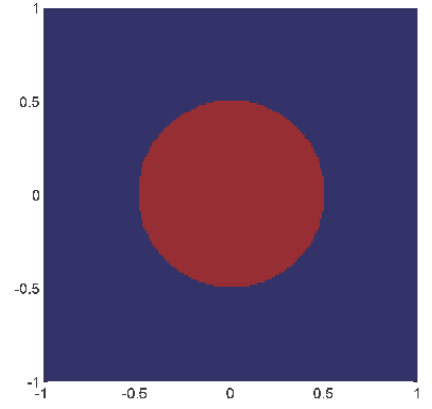

(a)

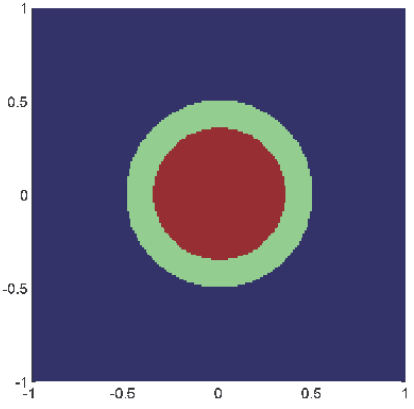

(b)

Figure 1: Configuration of the refractive index in a circular domain $D$ : homogeneous case (a), piecewise constant case (b).

In both cases the first transmission eigenvalue is analytically computable using the following result (that can be obtained using separation of variables).

Proposition 5.1. For a circle of radius $R$ and refractive index $n$, if $k_{0, \mathrm{TM}}^{*}$ and $k_{0, \mathrm{TE}}^{*}$ denote the first transmission eigenvalues respectively in the case of transverse magnetic and transverse electric polarized waves, then

$$
\begin{aligned}
& k_{0, \mathrm{TM}}^{*}=\min \left\{k \mid \exists p \in \mathbb{N} \text { s.t. } \operatorname{det}\left(A_{p, k}^{\mathrm{TM}}\right)=0\right\}, \\
& k_{0, \mathrm{TE}}^{*}=\min \left\{k \mid \exists p \in \mathbb{N} \text { s.t. } \operatorname{det}\left(A_{p, k}^{\mathrm{TE}}\right)=0\right\},
\end{aligned}
$$

with

$$
\begin{gathered}
A_{p, k}^{\mathrm{TM}}:=\left(\begin{array}{cc}
J_{p}(k R) & J_{p}(k \sqrt{n} R) \\
k J_{p-1}(k R)-\frac{p}{R} J_{p}(k R) & k \sqrt{n} J_{p-1}(k \sqrt{n} R)-\frac{p}{R} J_{p}(k \sqrt{n} R)
\end{array}\right), \\
A_{p, k}^{\mathrm{TE}}:=\left(\begin{array}{cc}
J_{p}(k R) & J_{p}(k \sqrt{n} R) \\
k J_{p-1}(k R)-\frac{p}{R} J_{p}(k R) & \frac{1}{\sqrt{n}}\left(k \sqrt{n} J_{p-1}(k \sqrt{n} R)-\frac{p}{R} J_{p}(k \sqrt{n} R)\right)
\end{array}\right) .
\end{gathered}
$$

Proposition 5.2. For a circle made of two concentric layers of external radius $R_{e}$ and internal radius $R_{i}$, if $n_{e}$ and $n_{i}$ respectively denote the refractive index of the exterior and interior layers and if $k_{0, \mathrm{TM}}^{*}$ and $k_{0, \mathrm{TE}}^{*}$ are the first transmission eigenvalues respectively in the case of transverse magnetic and transverse electric polarized waves, then

$$
\begin{aligned}
& k_{0, \mathrm{TM}}^{*}=\min \left\{k \mid \exists p \in \mathbb{N} \text { s.t. } \operatorname{det}\left(B_{p, k}^{\mathrm{TM}}\right)=0\right\}, \\
& k_{0, \mathrm{TE}}^{*}=\min \left\{k \mid \exists p \in \mathbb{N} \text { s.t. } \operatorname{det}\left(B_{p, k}^{\mathrm{TE}}\right)=0\right\},
\end{aligned}
$$

with

$$
\begin{gathered}
B_{p, k}^{\mathrm{TM}}:=\left(\begin{array}{cccc}
J_{p}\left(k R_{e}\right) & -J_{p}\left(k \sqrt{n_{e}} R_{e}\right) & -H_{p}^{(1)}\left(k \sqrt{n_{e}} R_{e}\right) & 0 \\
J_{p}^{\prime}\left(k R_{e}\right) & -J_{p}^{\prime}\left(k \sqrt{n_{e}} R_{e}\right) & -H_{p}^{(1)^{\prime}}\left(k \sqrt{n_{e}} R_{e}\right) & 0 \\
0 & J_{p}\left(k \sqrt{n_{e}} R_{i}\right) & H_{p}^{(1)}\left(k \sqrt{n_{e}} R_{i}\right) & -J_{p}\left(k \sqrt{n_{i}} R_{i}\right) \\
0 & J_{p}^{\prime}\left(k \sqrt{n_{e}} R_{i}\right) & H_{p}^{(1)^{\prime}}\left(k \sqrt{n_{e}} R_{i}\right) & -J_{p}^{\prime}\left(k \sqrt{n_{i}} R_{i}\right)
\end{array}\right), \\
B_{p, k}^{\mathrm{TE}}:=\left(\begin{array}{cccc}
J_{p}\left(k R_{e}\right) & -J_{p}\left(k \sqrt{n_{e}} R_{e}\right) & -H_{p}^{(1)}\left(k \sqrt{n_{e}} R_{e}\right) & 0 \\
J_{p}^{\prime}\left(k R_{e}\right) & -\frac{1}{n_{e}} J_{p}^{\prime}\left(k \sqrt{n_{e}} R_{e}\right) & -\frac{1}{n_{e}} H_{p}^{(1)^{\prime}}\left(k \sqrt{n_{e}} R_{e}\right) & 0 \\
0 & J_{p}\left(k \sqrt{n_{e}} R_{i}\right) & H_{p}^{(1)}\left(k \sqrt{n_{e}} R_{i}\right) & -J_{p}\left(k \sqrt{n_{i}} R_{i}\right) \\
0 & \frac{1}{n_{e}} J_{p}^{\prime}\left(k \sqrt{n_{e}} R_{i}\right) & \frac{1}{n_{e}} H_{p}^{(1)^{\prime}}\left(k \sqrt{n_{e}} R_{i}\right) & -\frac{1}{n_{i}} J_{p}^{\prime}\left(k \sqrt{n_{i}} R_{i}\right)
\end{array}\right) .
\end{gathered}
$$

Remark 5.1. Studying or plotting the behavior of $\operatorname{det}\left(A_{p, k}^{\mathrm{TM}}\right), \operatorname{det}\left(A_{p, k}^{\mathrm{TE}}\right), \operatorname{det}\left(B_{p, k}^{\mathrm{TM}}\right), \operatorname{det}\left(B_{p, k}^{\mathrm{TE}}\right)$ for $k \in \mathbb{R}$, one can observe that few $p \in \mathbb{N}$ (the firsts) are enough for determining $k_{0}^{*}$. 
Problem $P\left(D, k_{0}^{*}, n\right)$ is solved with $P_{1}$-finite elements based on the variational formulations 26 and (54). The discretization step is $\sim \frac{1}{10}$ of the wavelength inside the medium. The matrices are assembled using FreeFem++ [16] and the associate eigenvalue problem is solved in Matlab with the preset function eigs, in modality 'sm' (i.e. look for smaller magnitude eigenvalues). Since the eigenvalue problem formulation correspond with a generalized eigenvalue problem for non self-adjoint matrices and with degenerate mass matrices, there is no guarantee for convergence of the algorithm. Moreover, the mixed formulation is equivalent to the original one only under regularity assumptions on the solution. This is why parasite values are observed in the numerical computations of the eigenvalues. These parasite values correspond to solutions $u$ that do not satisfy the boundary condition $\partial u / \partial \nu=0$ on $\partial D$. From the practical point of view we observed that these bad values can simply be cut off by respecting the lower bound $n^{*}=\min \left\{-\lambda / k_{0}^{* 2}>1\right\}$ [3, 4.

Figure 2 shows results achievable when $D$ is a circle of radius 0.5 and has a constant refractive index. The value of $n$ is well recovered, with a better precision occurring for small $n$. As it is clear from Figure 2, with the increasing of the value of $n, k_{0}^{*}$ decreases, clustering around $k=1$. Since the computation of transmission eigenvalues is precise up to two digits, the previous consideration suggests that the difference in precision between reconstructions for small and large values of $n$ is due to a not enough precise approximation of $k_{0}^{*}$ for large $n$.
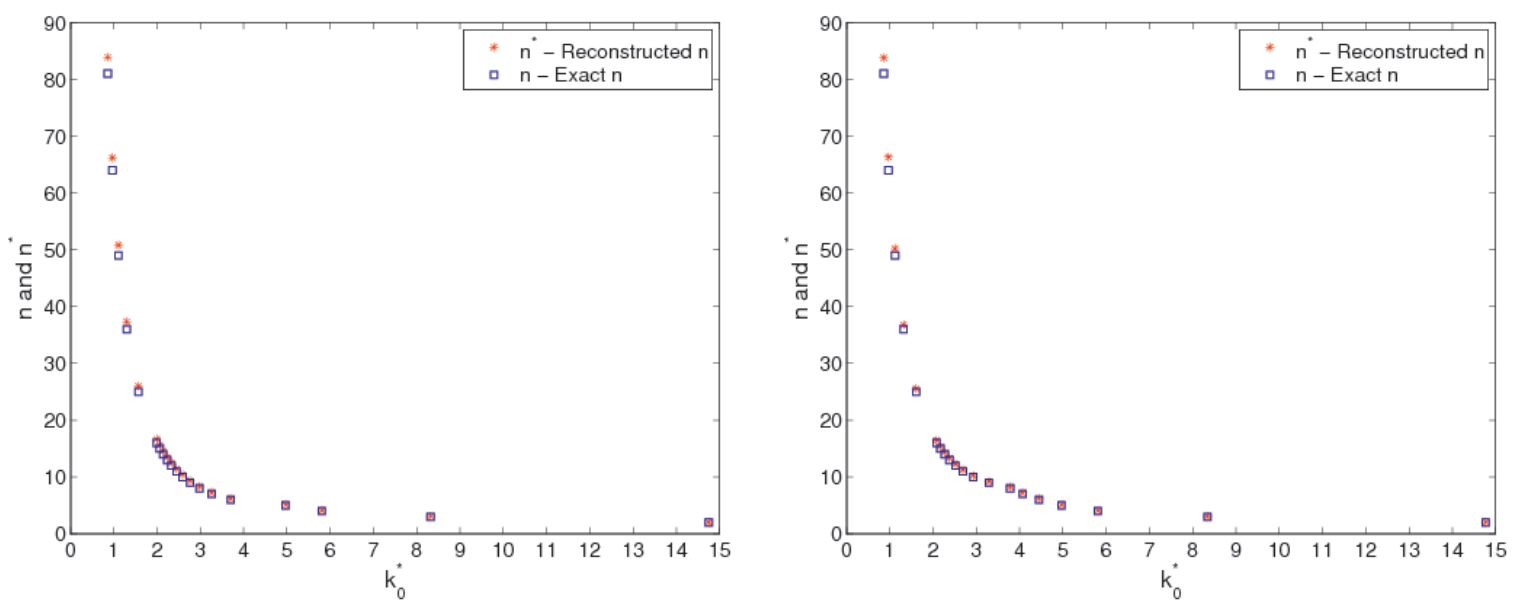

Figure 2: Reconstructed index for a homogeneous circle of radius 0.5. TM case (left) and TE case (right).

Figures 3 and 4 are associated with the two layered case where the areas of the two layers are equal $\left(R_{e}=0.5\right.$ and $\left.R_{i}=\frac{1}{2 \sqrt{2}}\right)$. The values of $n_{e}$ and $n_{i}$ are varied by maintaining the difference $n_{e}-n_{i}$ equals to 6 , (for example $\left.\left(n_{e}, n_{i}\right)=(8,2),(9,3), \ldots\right)$ for Figure 3 . As in previous figures, we plot the value of the reconstructed value $n^{*}$ of $n$ (y-axis) against the first transmission eigenvalue (x-axis); blue lines corresponding to the value of $n_{i}$ and $n_{e}$. They are over plotted in order to clarify wether or not $n^{*}$ fall in $\left[n_{i}, n_{e}\right]$. In Figure 4 the role of $n_{e}$ and $n_{i}$ are reversed to emphasize how a different structure (and not only different values of $n$ or different layer areas) could affect the reconstruction. 

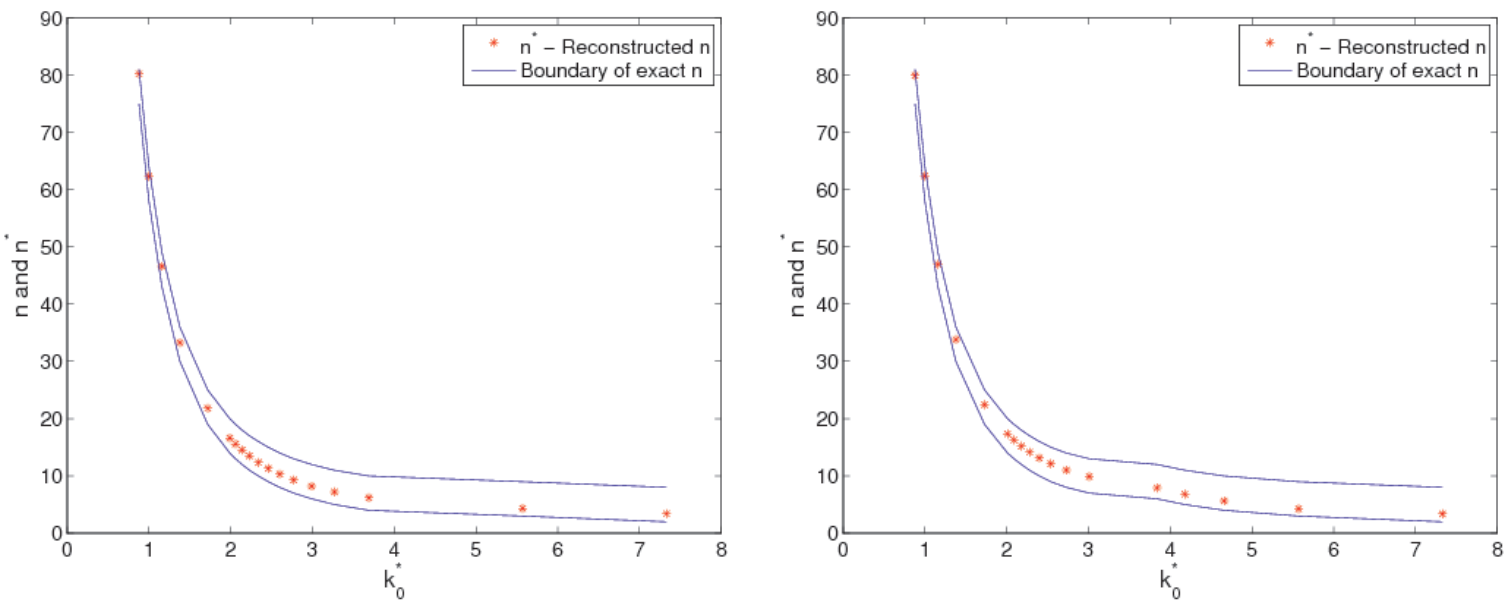

Figure 3: Reconstruction of the index in the case of a two layered circle $\left(R_{e}=0.5\right.$ and $\left.R_{i}=\frac{1}{2 \sqrt{2}}\right)$. The exact refractive index is such that $n_{e}-n_{i}=6$. The reconstructed value is shown by $*$ and the solid line indicate the exact values of $n_{e}$ and $n_{i}$. TM case (left) and TE case (right).
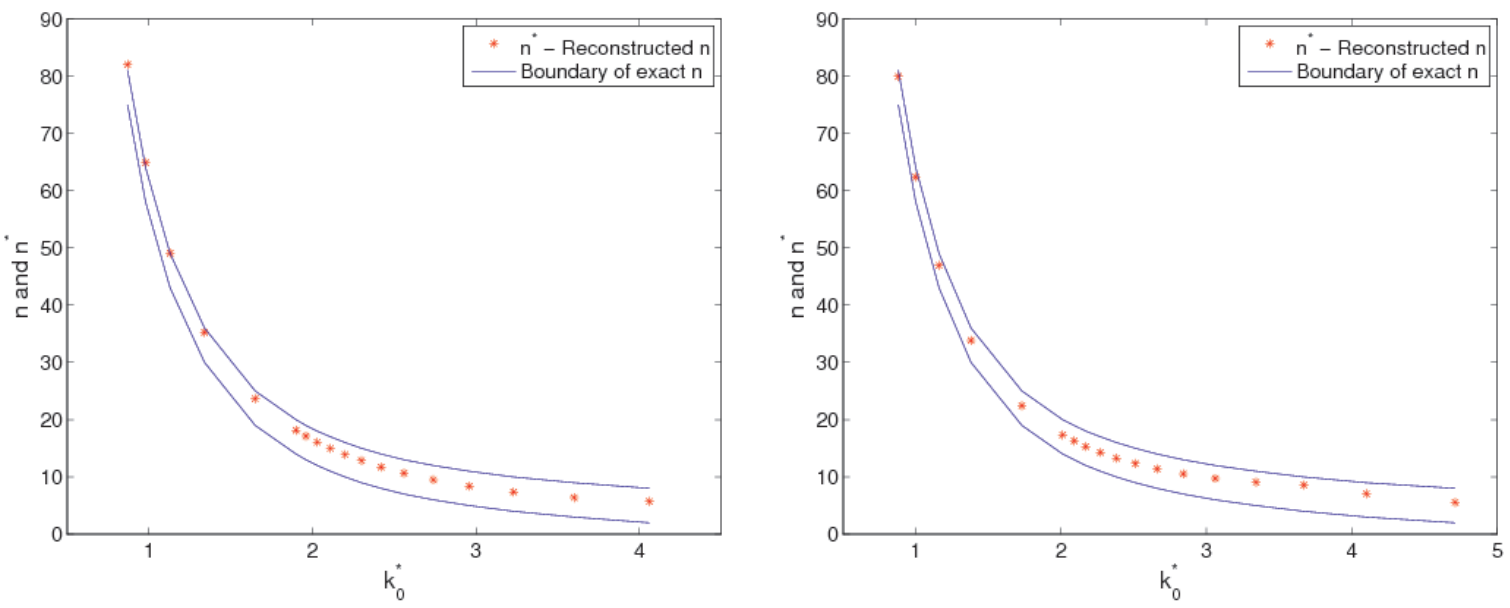

Figure 4: Same settings as in Figure 3 but with $n_{i}-n_{e}=6$. TM case (left) and TE case (right).

In the previous examples one observes that it is not simple to relate the value of the reconstructed constant index $n^{*}$ with non-constant refractive indexes. This is confirmed by next examples where we have not been able to observe a relation more accurate than

$$
\min _{x \in D} n(x) \leq n^{*} \leq \max _{x \in D} n(x)
$$

Understanding how transmission eigenvalues distribute depending on shape and dimension of the scatterer, on its index of refraction and on polarization of the wave, is, at present, still an open problem. The next few experiments are an overview of this complex relation between $n^{*}$ and different configurations of the material inside $D$ which is still a circle of radius 0.5.

With Figures 5 and 6. (respectively for TM and TE polarization), we want to investigate how different structures of $n$ would lead to different transmission eigenvalues (and consequently to different $n^{*}$ ) even if $\min _{D} n$ and $\max _{D} n$ are not modified. In both cases we consider a circular scatterer of radius 0.5 containing a concentric circular layer of varying radius. For fixed values of 
the refractive index $n_{i}=6$ and $n_{e}=12$ we show how $k_{0}^{*}$ and $n^{*}$ behave with respect to the radius of the internal circle $R_{i}$.
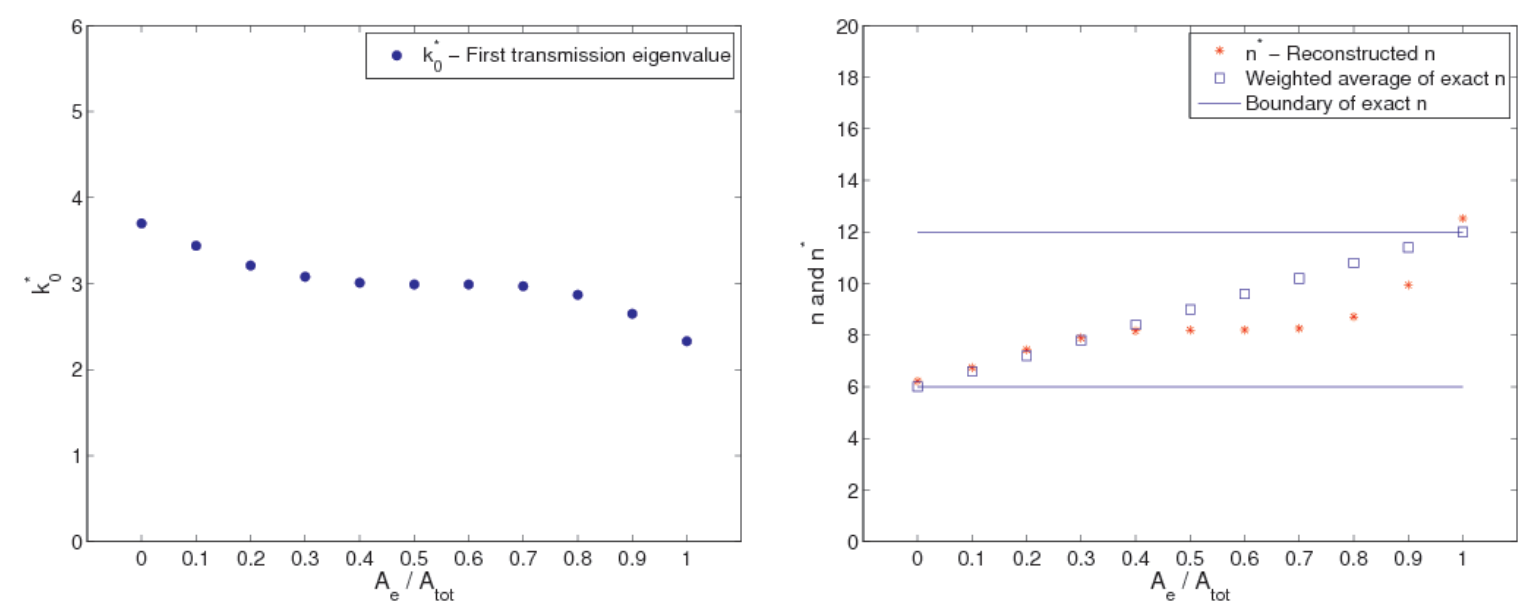

Figure 5: Results for TM polarized incident waves in the case of a two layered circle with $R_{e}=0.5$ and a varying $R_{i}$. The exact index values are $n_{i}=6$ and $n_{e}=12$. The x-axis represents the values of $A_{e} / A_{t o t}$, ratio of external layer area to the total circle area. Left: first transmission eigenvalue. Right: reconstructed $n^{*}$.

As expected, since the first transmission eigenvalue decreases with respect to the index of refraction [6], it should be increasing with respect to $R_{i}$ (since $n_{e}>n_{i}$ ) and therefore decreasing with respect to the area of the external layer $A_{e}$. However, the dependence is completely nonlinear. The values of $n^{*}$ have reversed monotonicity property with respect to the first transmission eigenvalue and therefore increases with $A_{e}$. The behavior of $n^{*}$ is also nonlinear as demonstrated by the comparison with the weighted average of the exact $n$.
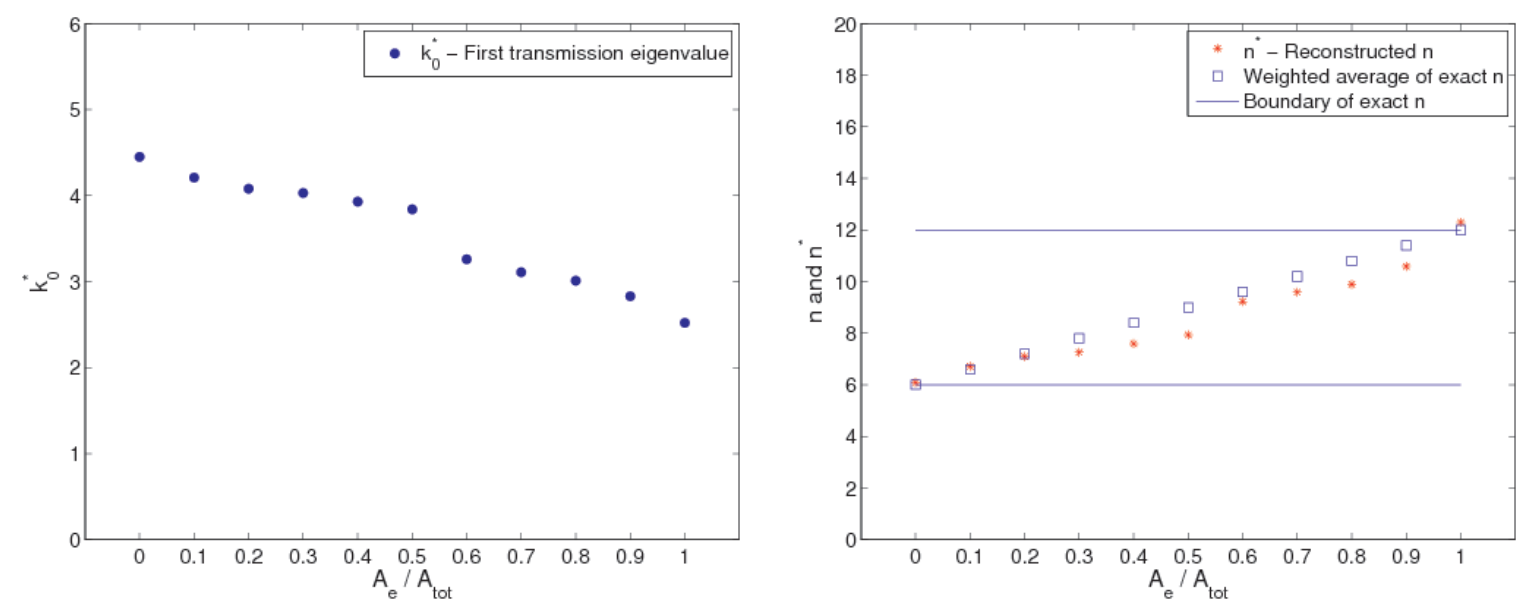

Figure 6: Results for TE polarized incident waves in the case of a two layered circle with $R_{e}=0.5$ and a varying $R_{i}$. The exact index values are $n_{i}=6$ and $n_{e}=12$. The x-axis represents the values of $A_{e} / A_{t o t}$, ratio of external layer area to the total circle area. Left: first transmission eigenvalue. Right: reconstructed $n^{*}$. 


\subsection{Numerical results for cases where the geometry is not known a priori}

In this last section we present some numerical experiments and results that are related to a more realistic inverse scattering experiment: i.e. a situation where both the geometry $D$ of the scatterer and its refractive index $n$ are unknown and the only data at our disposal are the farfields associated with many incident directions. Since in the previous section it has been shown that the method performance for TM polarized waves and for TE polarized waves is similar, we show only simulations for TM waves. The inversion algorithm is the following:

- Apply the LSM algorithm (as described in Section 2) to find an approximation $\tilde{D}$ of the shape of $D$.

- Determine the smallest transmission eigenvalue $k_{0}^{*}$ (for each connected component of $D$ ) using the method described in Section 3.

- Solve the eigenvalue problem $P\left(\tilde{D}, k_{0}^{*}, n\right)$ to obtain a constant approximation $n^{*}$ of the exact index $n$ inside $D$.

Following this scheme, for each example, we shall present both the LSM reconstruction and the graph of $k \mapsto\left\|g_{z}\right\|_{L^{2}(\Omega)}$ for several $z \in \tilde{D}$. Simulations are done using 20 emitting antennas, 20 measuring antennas and by corrupting the data with $5 \%$ random noise.

Figures are collected in panels (Figures 7-13) and the values of the corresponding constant approximation of $n$ are indicated in Tables $1-7$.

\subsubsection{The case of circular geometries.}

In this case comparisons can be made with the exact calculations made in previous section for transmission eigenvalues. It has also the advantage of allowing fast computation of the Green function for inhomogeneous (layered) backgrounds and fast implementation of LSM (see Appendix).

The first example corresponds with the two layered configuration of $D$ (shown in Figure 1 . right) with an external radius 0.5 and an internal radius 0.354 , embedded in the vacuum where we considered four configurations for the indexes of refraction $n=n_{e}$ for the exterior layer and $n=n_{i}$ for the interior layer. Figure 7 f(a1-a2) shows the LSM reconstruction for $n_{e}=22$ and $n_{i}=19$; for four different configurations of the index of refraction $\left(n_{e}=8, n_{i}=8\right),\left(n_{e}=11, n_{i}=5\right)$, $\left(n_{e}=22, n_{i}=19\right)$ and $\left(n_{e}=67, n_{i}=61\right)$, we present $k \mapsto\left\|g_{z}\right\|_{L^{2}(\Omega)}$ that allows us to determine the first transmission eigenvalue. The corresponding values of $n^{*}$ are collected in Table 1 . 


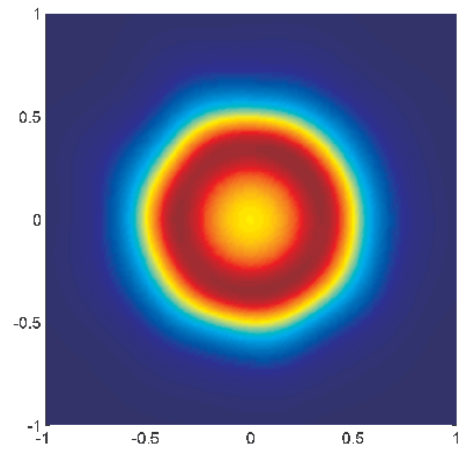

$\left(a_{1}\right)$

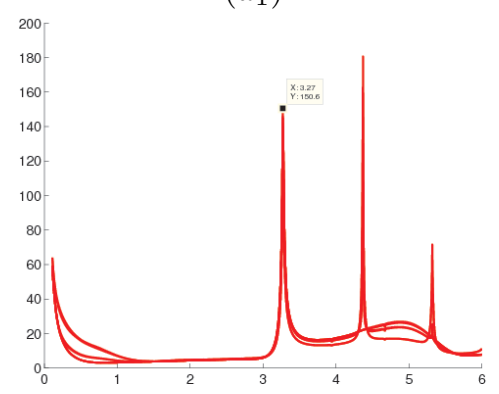

$\left(b_{1}\right)$

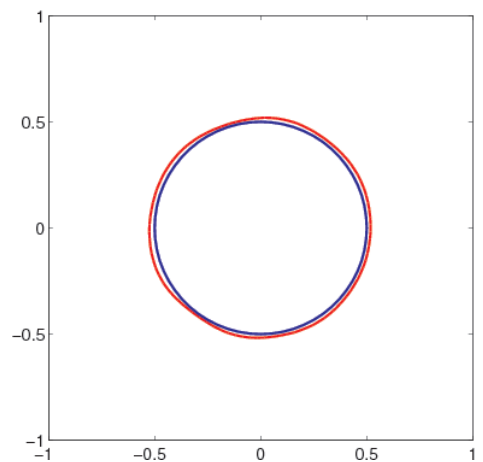

$\left(a_{2}\right)$

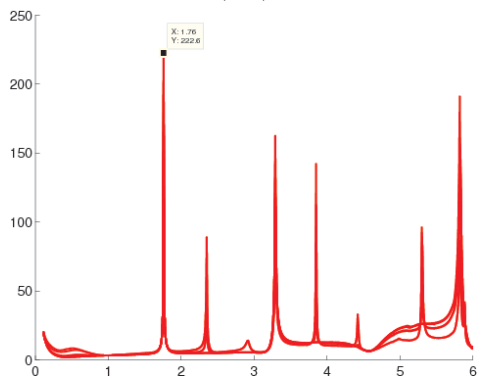

$\left(b_{2}\right)$

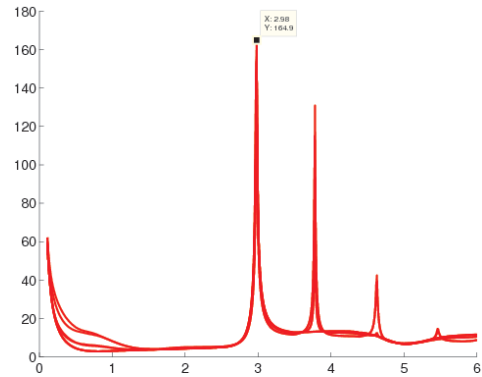

$\left(a_{3}\right)$

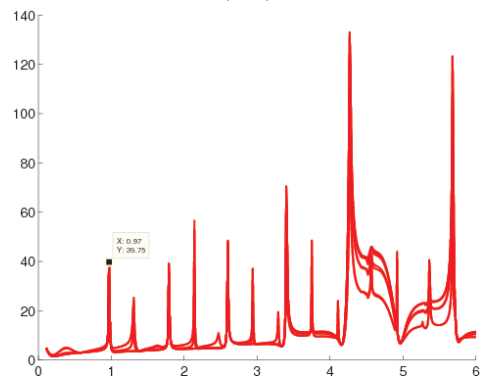

$\left(b_{3}\right)$

Figure 7: Two layered circular inclusion embedded in the vacuum (Figure 1-right). $\left(a_{1}\right)$ LSM reconstruction at $k=4.2, n_{e}=22$ and $n_{i}=19$. $\left(a_{2}\right)$ reconstructed shape (red) versus exact shape (blue). $\left(a_{3}\right),\left(b_{1}\right),\left(b_{2}\right),\left(b_{3}\right)$ plots of $k \mapsto\left\|g_{z}\right\|_{L^{2}(\Omega)}$ for several choices of $z$, respectively for $\left(n_{e}, n_{i}\right)=(8,8),(11,5),(22,19),(67,61)$.

\begin{tabular}{|l|l|l|l|l|}
\hline$n_{e}$ & $n_{i}$ & $k_{0}^{*}$ & $n^{*}$ - exact shape & $n^{*}$-reconstructed shape \\
\hline 8 & 8 & 2.98 & 8.07 & 7.61 \\
11 & 5 & 3.27 & 7.05 & 6.69 \\
22 & 19 & 1.76 & 20.28 & 18.86 \\
67 & 61 & 0.97 & 64.11 & 59.42 \\
\hline
\end{tabular}

Table 1: Transmission eigenvalues and reconstructed index associated with Figure 7.

Figures 8, 9 and corresponding Tables 2, 3, describe the results achievable when the two layered inclusion of previous experiment is now embedded in an inhomogeneous background. The inhomogeneous background consists into a circular domain of radius 0.75 and with refractive index $n_{b}$ embedded in the vacuum.

Figure 8 and Table 2 show how the method perform for objects embedded in a non-absorbing background. As expected, we observe that the transmission eigenvalues are different from those corresponding to the homogeneous background but the estimates $n^{*}$ are roughly the same. 


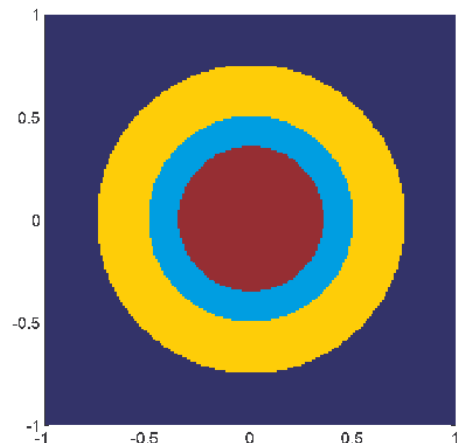

$\left(a_{1}\right)$

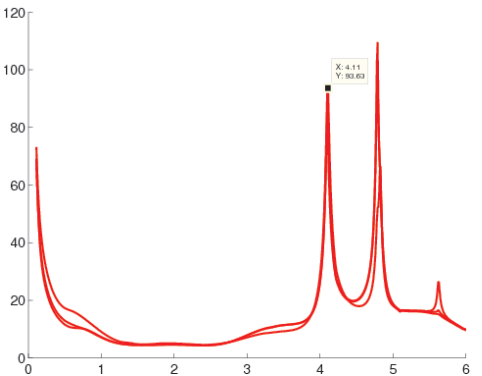

$\left(b_{1}\right)$

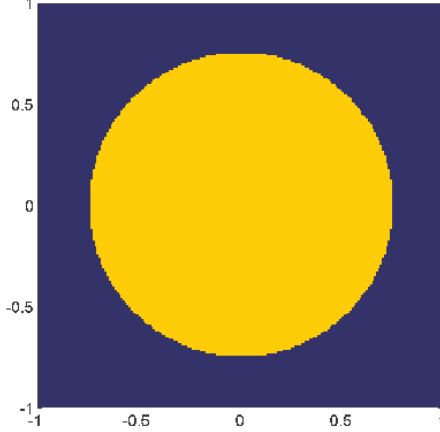

$\left(a_{2}\right)$

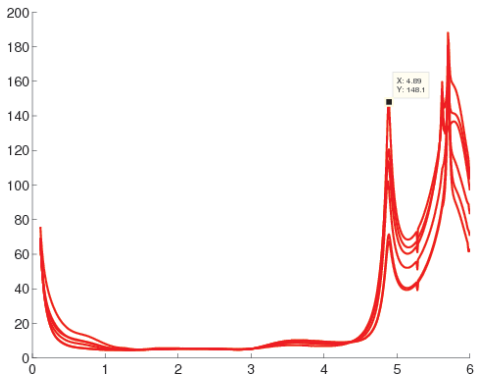

$\left(b_{2}\right)$

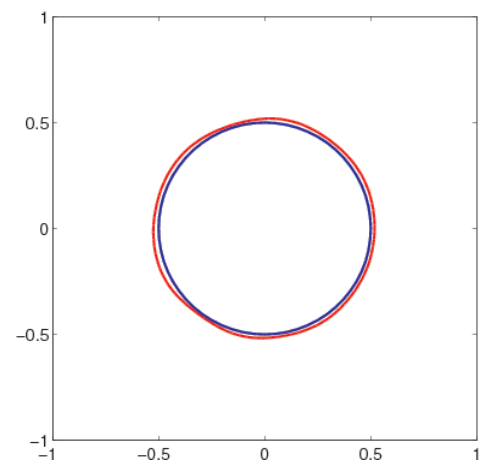

$\left(a_{3}\right)$

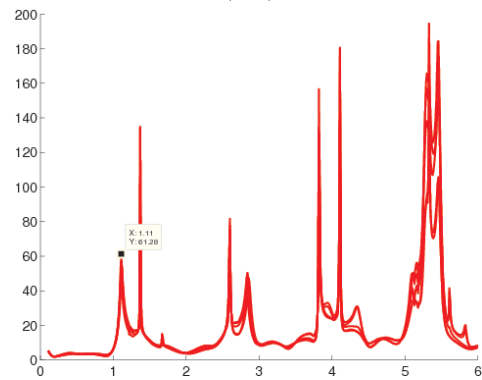

$\left(b_{3}\right)$

Figure 8: Two layered circular inclusion $\left(a_{1}\right)$ embedded in an inhomogeneous background $\left(a_{2}\right)$. $\left(a_{3}\right)$ : reconstructed shape (red) versus exact shape (blue). $\left(b_{1}\right),\left(b_{2}\right),\left(b_{3}\right)$ : plots of $k \mapsto\left\|g_{z}\right\|_{L^{2}(\Omega)}$ for several choices of $z$, respectively for $\left(n_{e}, n_{i}, n_{b}\right)=(8,8,2),(11,5,2),(67,61,10)$.

\begin{tabular}{|l|l|l|l|l|l|}
\hline$n_{e}$ & $n_{i}$ & $n_{b}$ & $k_{0}^{*}$ & $n^{*}$ - exact shape & $n^{*}$-reconstructed shape \\
\hline 8 & 8 & 2 & 4.11 & 8.09 & 7.70 \\
11 & 5 & 2 & 4.89 & 6.92 & 5.04 \\
67 & 61 & 10 & 1.11 & 64.35 & 61.44 \\
\hline
\end{tabular}

Table 2: Transmission eigenvalues and reconstructed index associated with Figure 8

Figure 9 and Table 3 correspond with the case of an absorbing background. We develop here the idea explained in Section 3.3.1 and show that, when an artificial non-absorbing background of index $\tilde{n}_{b}$ is used inside the reconstructed domain, real transmission eigenvalues can be computed, allowing to find roughly the same values of $n^{*}$ as in the non absorbing case. We remark that in this configuration the accuracy of $\tilde{D}$ (approximation of the shape of $D$ ) has a stronger influence on the accuracy of $n^{*}$ since it affects the computation of the incident field for the background. The latter is used in computing $\left\|g_{z}\right\|_{L^{2}(\Omega)}$ and therefore affect the precision of the first transmission eigenvalue. 


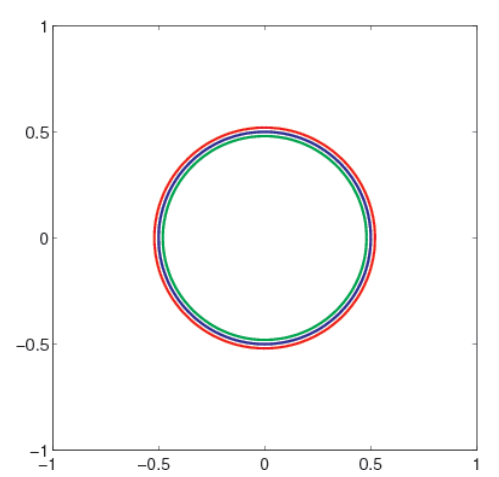

$\left(a_{1}\right)$

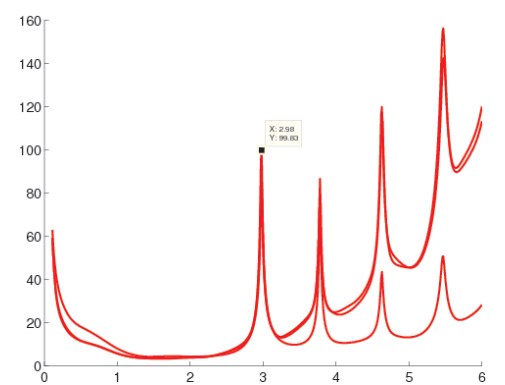

$\left(b_{1}\right)$

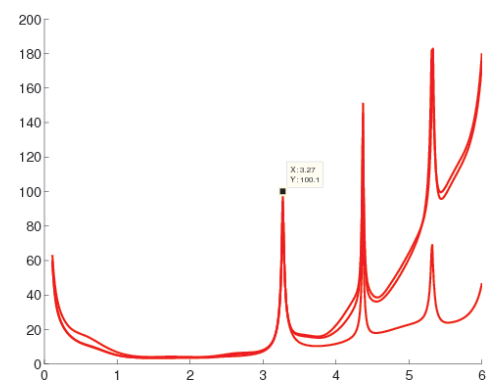

$\left(c_{1}\right)$

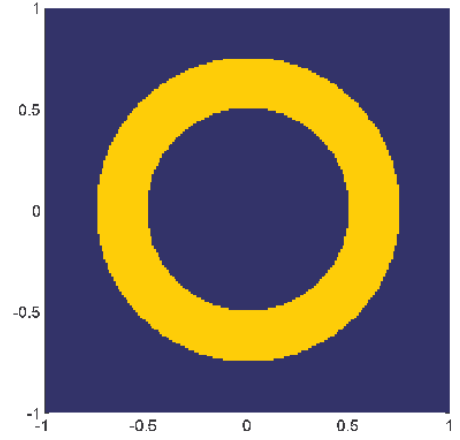

$\left(a_{2}\right)$

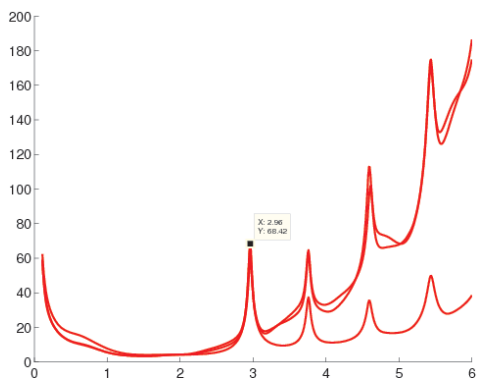

$\left(b_{2}\right)$

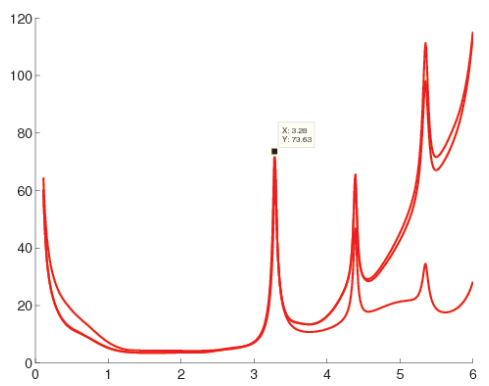

$\left(c_{2}\right)$

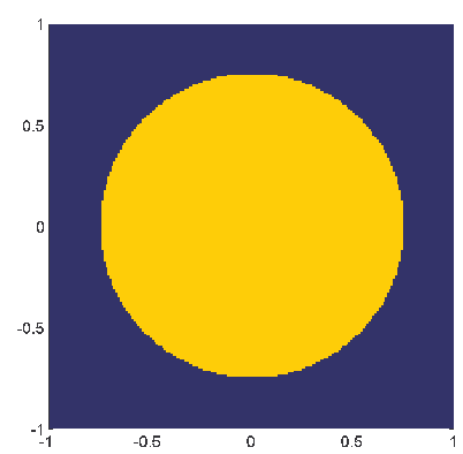

$\left(a_{3}\right)$

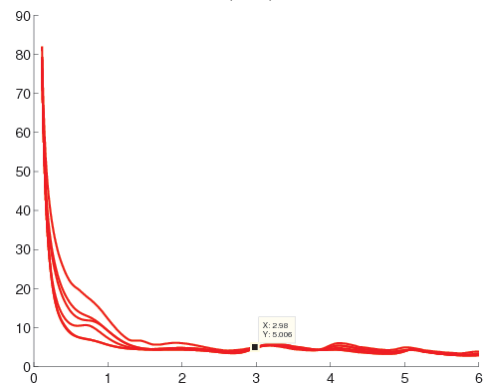

$\left(b_{3}\right)$

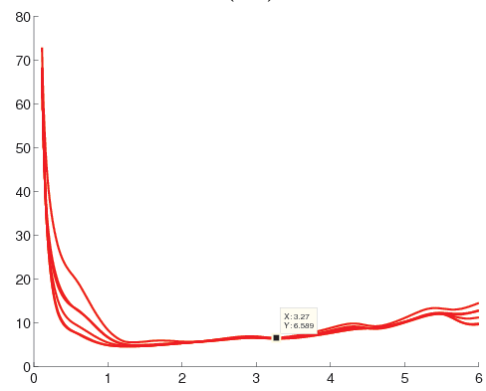

$\left(c_{3}\right)$

Figure 9: Two layered circular inclusion $\left(n_{e}, n_{i}\right)=(8,8)$, embedded in an inhomogeneous absorbing background $\left(a_{3}\right)$ with $n_{b}=2+\imath$. $\left(a_{1}\right)$ : reconstructed shape (red and green) versus exact shape (blue). $\left(a_{2}\right)$ : considered artificial background with $\tilde{n}_{b}=1$ inside $D$. $\left(b_{1}\right),\left(b_{2}\right),\left(b_{3}\right)$ : plots of $k \mapsto\left\|g_{z}\right\|_{L^{2}(\Omega)}$ for several choices of $z$, respectively for the exact shape and artificial background, reconstructed shape (red) and artificial background, exact shape and true background. $\left(c_{1}\right),\left(c_{2}\right)$, $\left(c_{3}\right)$ : same as $\left(b_{1}\right),\left(b_{2}\right),\left(b_{3}\right)$ but for the case $\left(n_{e}, n_{i}\right)=(11,5)$ and for the green reconstructed shape.

\begin{tabular}{|l|l|l|l|l|l|}
\hline$n_{e}$ & $n_{i}$ & $n_{b}$ & $\tilde{n}_{b}$ & $\left(k_{0}^{*}, n^{*}\right)$ - exact shape & $\left(k_{0}^{*}, n^{*}\right)$ - reconst. shape \\
\hline 8 & 8 & $2+\imath$ & 1 & $(2.98,8.07)$ & $(2.96,7.69)$ \\
\hline 11 & 5 & $2+\imath$ & 1 & $(3.27,7.05)$ & $(3.28,7.43)$ \\
\hline
\end{tabular}

Table 3: Transmission eigenvalues and reconstructed index associated with Figure 9 


\subsubsection{Cases of non circular geometries}

In Figure 10 and Figure 11 the case of scatterers that are not circular is investigated. In both figures a two layered inclusion is considered. The exact profile of $D$, the LSM reconstruction for $n_{e}=22, n_{i}=19$ and transmission eigenvalues for $\left(n_{e}, n_{i}\right)=(8,8),(11,5),(22,19)$ are respectively shown for a layered ellipse and for an ellipse embedded into a rectangle. Tables 4 and 5 present the values of $n^{*}$ achievable using the values of $k_{0}^{*}$ and the extracted profile $\tilde{D}$. One can draw roughly similar conclusions as in the case of circular geometries.

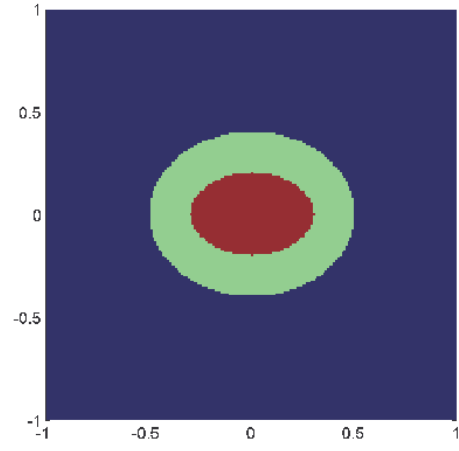

$\left(a_{1}\right)$

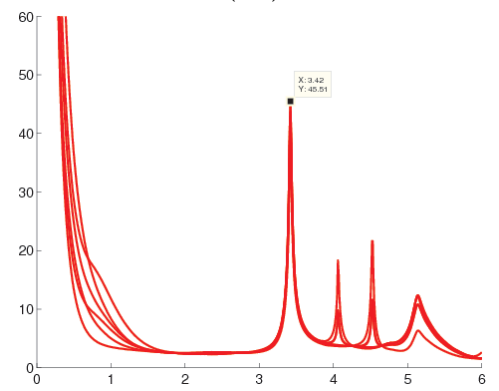

$\left(b_{1}\right)$

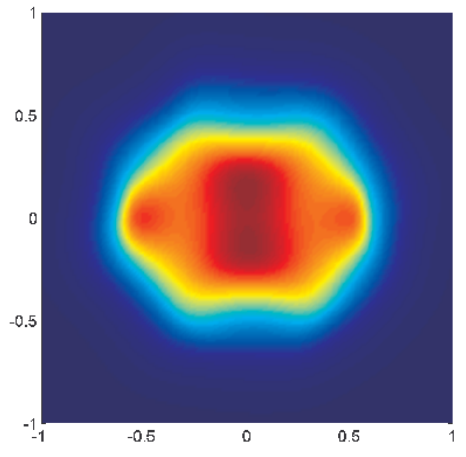

$\left(a_{2}\right)$

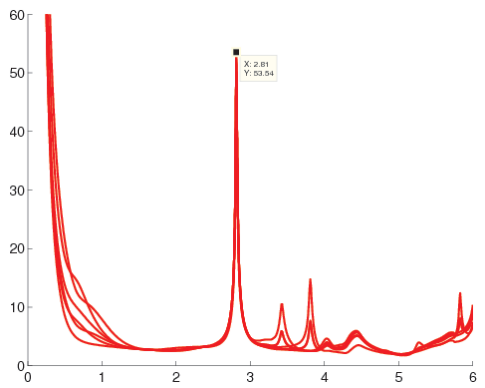

$\left(b_{2}\right)$

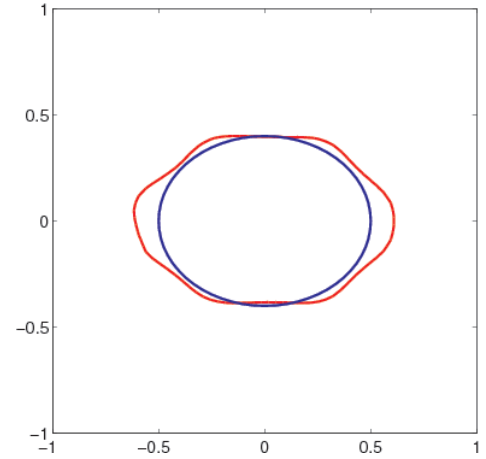

$\left(a_{3}\right)$

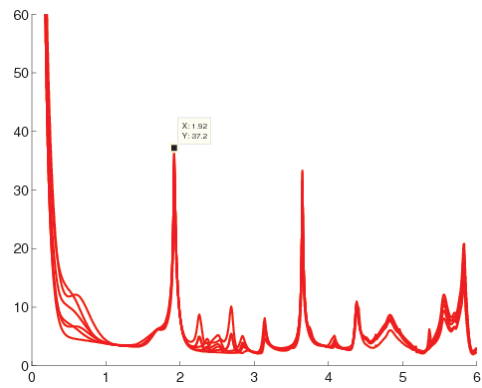

$\left(b_{3}\right)$

Figure 10: Two layered ellipsoidal inclusion $\left(a_{1}\right)$ embedded in the vacuum. $\left(a_{2}\right)$ : LSM reconstruction for $k=4.16, n_{e}=22$ and $n_{i}=19$. $\left(a_{3}\right)$ : reconstructed shape (red) versus exact shape (blue). $\left(b_{1}\right),\left(b_{2}\right),\left(b_{3}\right)$ : plots of $k \mapsto\left\|g_{z}\right\|_{L^{2}(\Omega)}$ for several choices of $z$, respectively for $\left(n_{e}, n_{i}\right)=(8,8),(11,5),(22,19)$.

\begin{tabular}{|l|l|l|l|l|}
\hline$n_{e}$ & $n_{i}$ & $k_{0}^{*}$ & $n^{*}-$ exact shape & $n^{*}$ - reconstructed shape \\
\hline 8 & 8 & 3.42 & 8.08 & 7.74 \\
11 & 5 & 2.81 & 11.10 & 10.50 \\
22 & 19 & 1.92 & 22.16 & 20.73 \\
\hline
\end{tabular}

Table 4: Transmission eigenvalues and reconstructed index associated with Figure 10. 


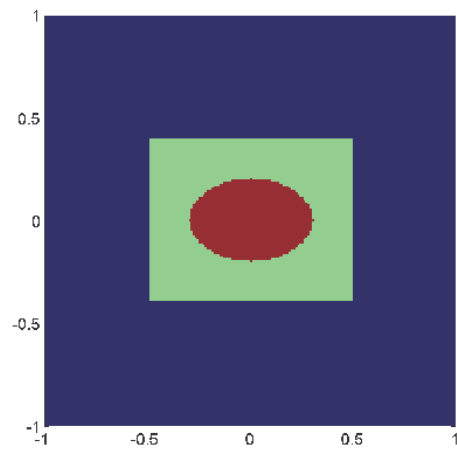

$\left(a_{1}\right)$

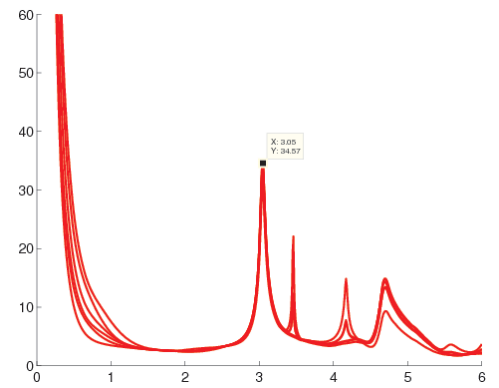

$\left(b_{1}\right)$

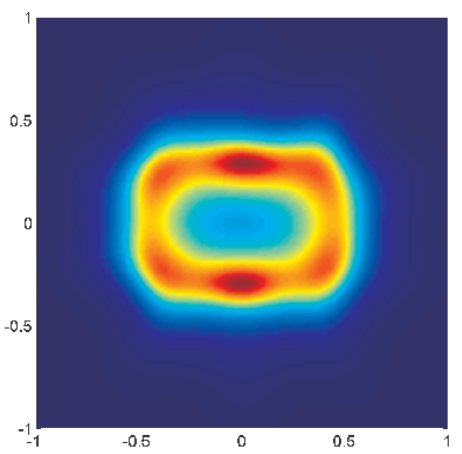

$\left(a_{2}\right)$

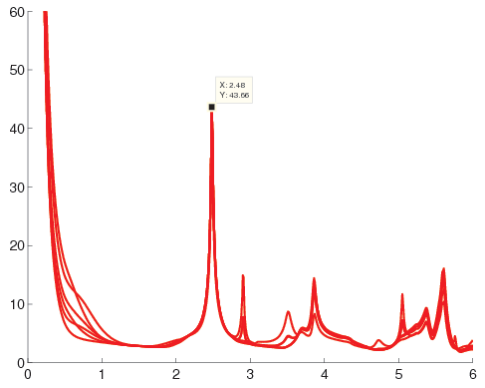

$\left(b_{2}\right)$

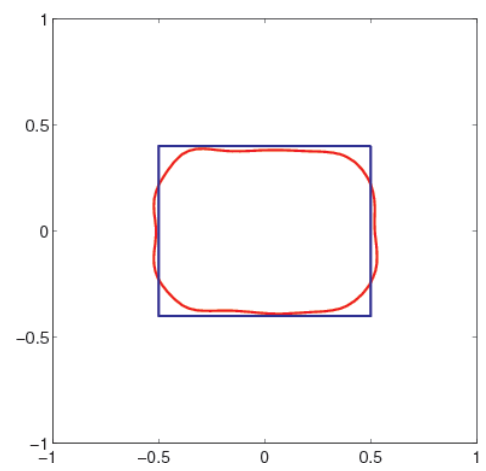

$\left(a_{3}\right)$

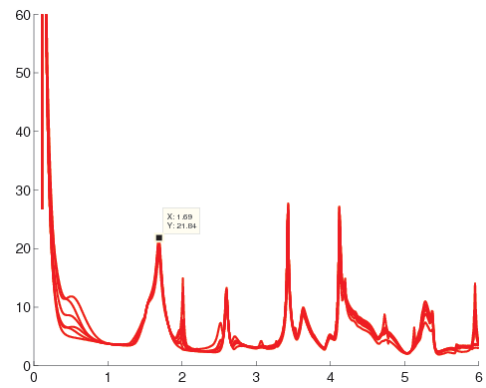

$\left(b_{3}\right)$

Figure 11: Rectangular inclusion containing an ellipsoidal layer $\left(a_{1}\right)$ embedded in the vacuum. $\left(a_{2}\right)$ : LSM reconstruction for $k=5.64, n_{e}=22$ and $n_{i}=19$. ( $\left.a_{3}\right)$ : reconstructed shape (red) versus exact shape (blue). $\left(b_{1}\right),\left(b_{2}\right),\left(b_{3}\right)$ : plots of $k \mapsto\left\|g_{z}\right\|_{L^{2}(\Omega)}$ for several choices of $z$, respectively for $\left(n_{e}, n_{i}\right)=(8,8),(11,5),(22,19)$.

\begin{tabular}{|l|l|l|l|l|}
\hline$n_{e}$ & $n_{i}$ & $k_{0}^{*}$ & $n^{*}$ - exact shape & $n^{*}$ - reconstructed shape \\
\hline 8 & 8 & 3.05 & 8.94 & 9.37 \\
11 & 5 & 2.48 & 12.62 & 13.27 \\
22 & 19 & 1.69 & 25.53 & 26.90 \\
\hline
\end{tabular}

Table 5: Transmission eigenvalues and reconstructed index associated with Figure 11.

\subsubsection{Cases of multiply connected inclusions}

Finally, inspired by Corollary 3.1, we present a simulation for a scatterer consisting in two distinct objects of different refractive indexes. Figures 12 and 13 jointly with Tables 6 and 7 prove that a reconstruction of both indexes of refraction is effectively achievable by considering $k \mapsto\left\|g_{z}\right\|_{L^{2}(\Omega)}$ first for several $z$ in one connected component and second for several $z$ in the other connected component. As one cas observe in part $\left(b_{1}\right)$ of Figure 13 small peaks are present in $k \mapsto\left\|g_{z}\right\|_{L^{2}(\Omega)}$ preceding the first transmission eigenvalue. A closer look reveals that those peaks correspond with the transmission eigenvalues of the other connected component (see part $\left(b_{3}\right)$ ). We think that this is due to the fact that the two connected components are close to each others. For instance, such perturbation is not observed in the case of Figure 12 where the distance between the two connected components is larger. 


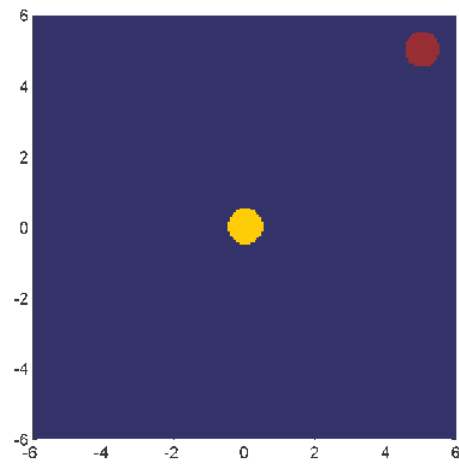

$\left(a_{1}\right)$

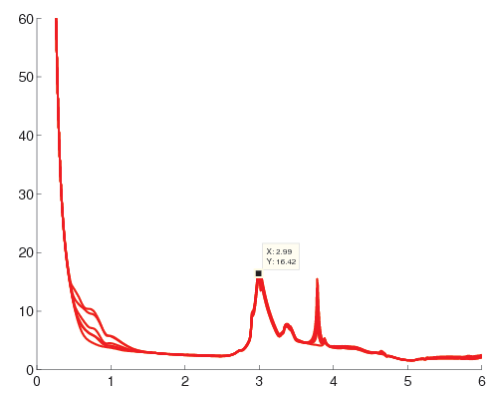

$\left(b_{1}\right)$

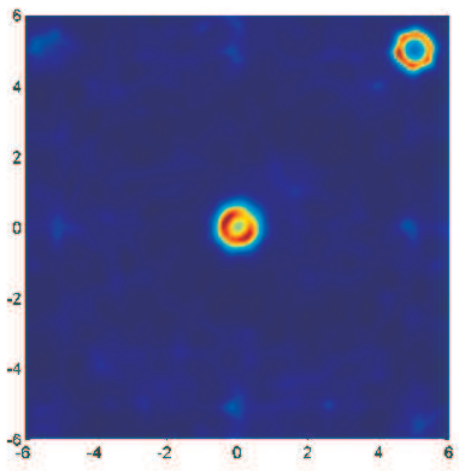

$\left(a_{2}\right)$

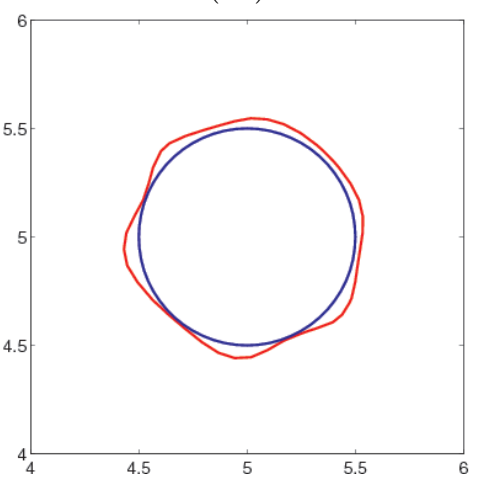

$\left(b_{2}\right)$

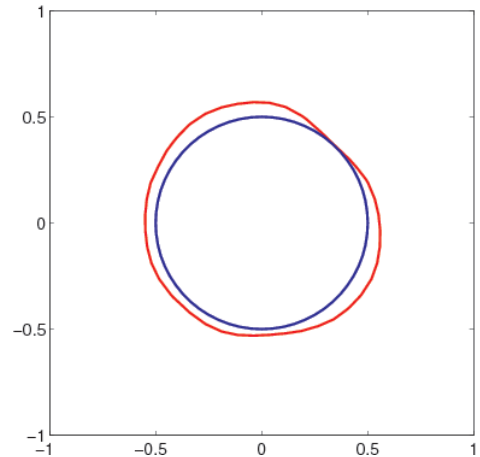

$\left(a_{3}\right)$

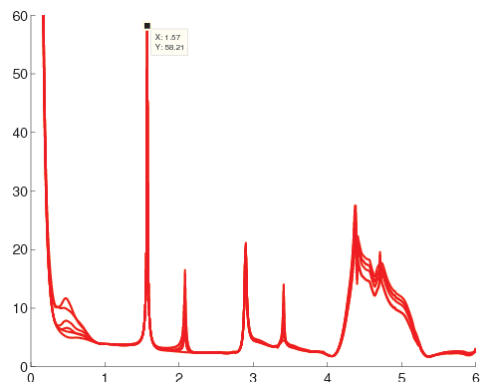

$\left(b_{3}\right)$

Figure 12: Two homogeneous circular inclusions $\left(a_{1}\right)$ : a circle $c_{1}$ centered at $(0,0)$ of refractive index $n=8$ and a circle $c_{2}$ centered at $(5,5)$ of refractive index $n=25$. $\left(a_{2}\right)$ : LSM reconstruction for $k=4.2$. $\left(a_{3}\right)$ and $\left(b_{2}\right)$ : reconstructed shape (red) versus exact shape (blue) respectively for $c_{1}$ and $c_{2}$. $\left(b_{1}\right)$ and $\left(b_{3}\right)$ : plots of $k \mapsto\left\|g_{z}\right\|_{L^{2}(\Omega)}$ for several choices of $z$, respectively for $c_{1}$ and $c_{2}$.

\begin{tabular}{|l|l|l|l|l|}
\hline circle & $n$ & $k_{0}^{*}$ & $n^{*}$ - exact shape & $n^{*}$ - reconstructed shape \\
\hline$c_{1}$ & 8 & 2.99 & 8.03 & 7.17 \\
$c_{2}$ & 25 & 1.57 & 25.17 & 24.60 \\
\hline
\end{tabular}

Table 6: Transmission eigenvalues and reconstructed index associated with Figure 12 .

\section{A Fast implementation of LSM in the case of a background with axisymmetric index}

We here explain a procedure that accelerates the LSM algorithm for inhomogeneous backgrounds that have piecewise constant and axisymmetric indexes. In this configuration, by exploiting the analytic expression of a solution to the Helmholtz equation (obtained by separation of variables) and by imposing a single regularization to the family of equations (7), we shall indicate how one can avoid a computation of the right hand sides for all sampling points, and therefore speed up the inversion of the data. Such a procedure can be useful in situations in which, for reasons related to the resolution power of the output or related to the material properties, we need to reconstruct the shape of the scatterer on a grid made of a large amount of points. We explain the procedure in the case of TM polarized wave (the case of TE case can be treated in a similar way). 


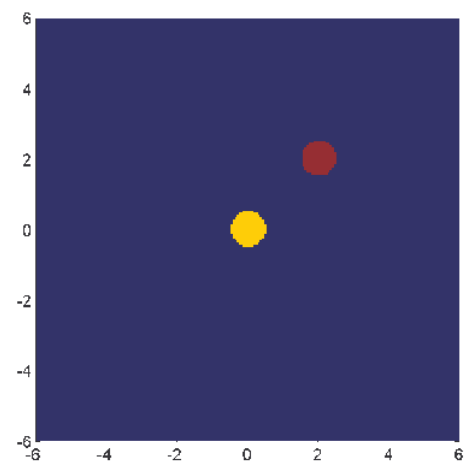

$\left(a_{1}\right)$

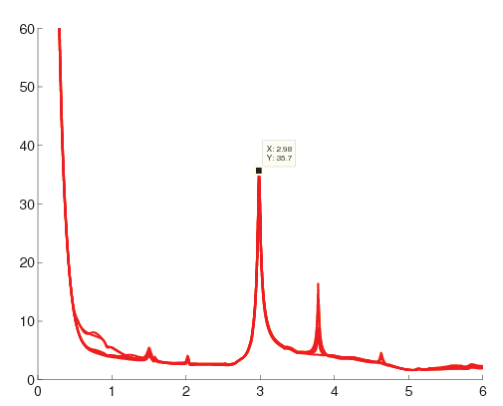

$\left(b_{1}\right)$

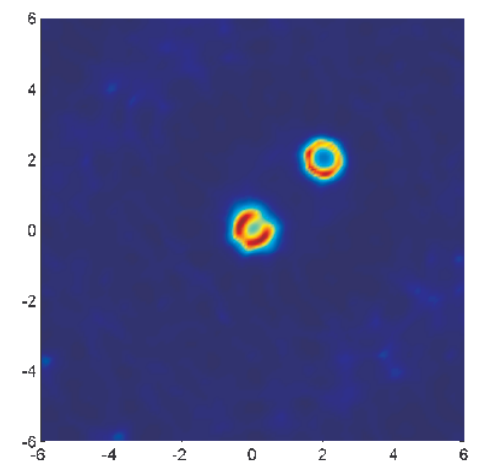

$\left(a_{2}\right)$

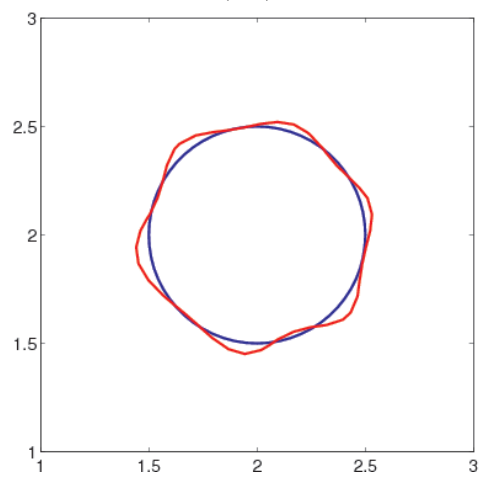

$\left(b_{2}\right)$

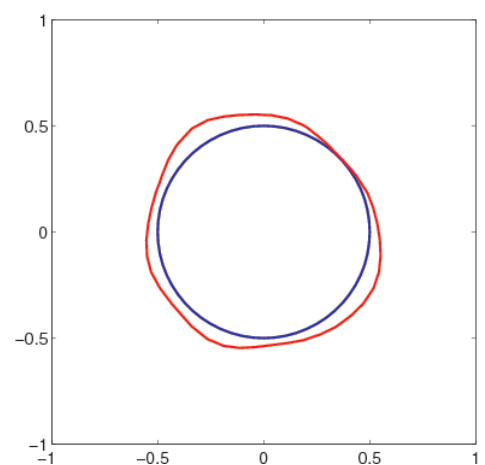

$\left(a_{3}\right)$

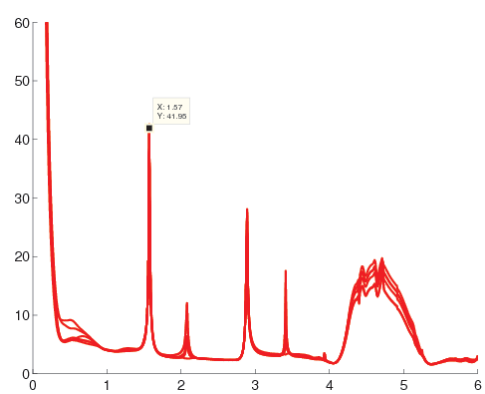

$\left(b_{3}\right)$

Figure 13: Two homogeneous circular inclusions $\left(a_{1}\right)$ : a circle $c_{1}$ centered at $(0,0)$ of refractive index $n=8$ and a circle $c_{2}$ centered at $(5,5)$ of refractive index $n=25 .\left(a_{2}\right)$ : LSM reconstruction for $k=4.2 .\left(a_{3}\right)$ and $\left(b_{2}\right)$ : reconstructed shape (red) versus exact shape (blue) respectively for $c_{1}$ and $c_{2}$. $\left(b_{1}\right)$ and $\left(b_{3}\right)$ : plots of $k \mapsto\left\|g_{z}\right\|_{L^{2}(\Omega)}$ for several choices of $z$, respectively for $c_{1}$ and $c_{2}$.

\begin{tabular}{|l|l|l|l|l|}
\hline circle & $n$ & $k_{0}^{*}$ & $n^{*}$ - exact shape & $n^{*}$ - reconstructed shape \\
\hline$c_{1}$ & 8 & 2.98 & 8.07 & 7.17 \\
$c_{2}$ & 25 & 1.57 & 25.17 & 24.60 \\
\hline
\end{tabular}

Table 7: Transmission eigenvalues and reconstructed index associated with Figure 13. 
In the case of incident plane waves, the solution to the Helmholtz equation in the case of piecewise constant and axisymmetric indexes is of the form

$$
u(x)=\sum_{m=-\infty}^{+\infty}\left[\alpha_{m}^{\ell} H_{m}^{(1)}\left(k r_{x} \sqrt{n(x)}\right)+\beta_{m}^{\ell} J_{m}\left(k r_{x} \sqrt{n(x)}\right)\right] \exp ^{i m \theta_{x}}
$$

in the region $\ell$ where $n(x)$ is constant, where $\left(r_{x}, \theta_{x}\right)$ are the polar coordinates of $x$ and where $H_{m}$ and $J_{m}$ are respectively Hankel and Bessel functions. The coefficients $\alpha_{m}^{\ell}$ and $\beta_{m}^{\ell}$ only depend on the incident field and on the geometry. If $\ell=0$ denotes the outer domain (vacuum) and $\ell=L$ denotes the inner homogeneous domain then $\alpha_{m}^{L}=0$ and $\beta_{m}^{0}=0$ correspond to the expansion of the incident field in the neighborhood of the outer boundary. More precisely, using the Jacobi-Anger expansion [8], the incident field is expanded as

$$
u^{i}(x)=\sum_{m=-\infty}^{+\infty} \beta_{m}^{0} J_{m}\left(k r_{x}\right) \exp ^{i m \theta_{x}}
$$

The remaining coefficients in expansion 70 are determined by imposing the continuity of the total field and of its normal derivative on the interfaces. One obtains for each harmonic $m$ a system of $2 L$ equations for $2 L$ unknowns. The matrix of the obtained system is independent of the right hand side. This enables for instance a quick evaluation of the background farfields (see (17)) since

$$
u_{\infty, b}^{s}(\hat{x}, d)=\sum_{m=-\infty}^{+\infty} \alpha_{m}^{0}(d) \frac{\sqrt{2}}{\pi} \exp ^{\frac{-i \pi}{2}(2 m+1)} \exp ^{i m \theta_{\hat{x}}} .
$$

One can also use this technique to evaluate the background Green tensor. In that case, $G(\cdot, z)$ has similar expression as $(70)$ in the layers that does not contain $z$ with the additional condition that $\beta_{m}^{0}=0$. In the layer that contain $z$ we add to that expression $\frac{i}{4} H_{0}^{(1)}(k \sqrt{n(x)}|x-z|)$. Using the addition formula in order to expand $x \mapsto \frac{i}{4} H_{0}^{(1)}(k \sqrt{n(x)}|x-z|)$ as a Fourier series one ends up, as in the previous case, with a $2 L \times 2 L$ system to solve for each harmonic or order $m$, with a matrix independent of the point $z$. We therefore infer an expression of $G_{\infty}$ (see 177$)$ of the form

$$
G_{\infty}(\hat{x}, z)=\sum_{m=-\infty}^{+\infty} \xi_{m}^{0}(z) \frac{\sqrt{2}}{\pi} \exp ^{\frac{-i \pi}{2}(2 m+1)} \exp ^{i m \theta_{\hat{x}}}
$$

The linear sampling method requires the resolution of

$$
F g_{z}=G_{\infty, z}
$$

for each $z$ point of $\mathbb{R}^{2}$ belonging to a grid containing the scatterer and where $F$ is defined in (17). If $F^{*}$ denotes the adjoint of $F$, the Tikhonov regularization of (74) is

$$
\left(F^{*} F+\alpha_{z} I\right) g_{z}=F^{*} G_{\infty, z}
$$

which has to be solved for each $z$ belonging to a grid containing the unknown object. Supposing $\alpha_{z}$ independent from $z$, i.e. supposing to choose the regularization parameter only once during all the reconstruction process (for instance, in [1] a no sampling approach to the linear sampling method is proposed and the problem of properly choosing $\alpha$ is analyzed), some simplification inside the far field equation is possible. In fact, defining

$$
\psi_{m}(x):=\frac{\sqrt{2}}{\pi} \exp ^{\frac{-i \pi}{2}(2 m+1)} \exp ^{i m \theta_{x}}
$$

we have that $\forall \hat{x} \in \Omega$

$$
G_{\infty}(\hat{x}, z)=\sum_{m=-M}^{M} \xi_{m}^{0}(z) \frac{\sqrt{2}}{\pi} \exp ^{\frac{-i \pi}{2}(2 m+1)} \exp ^{i m \theta_{\hat{x}}}=\sum_{m=-M}^{M} \xi_{m}^{0}(z) \psi_{m}\left(r, \theta_{\hat{x}}\right),
$$

RR $\mathrm{n}^{\circ} 7729$ 
assuming that we truncate the infinite series at $M \in \mathbb{N}$. The solution $g_{z}$ can be expanded as

$$
g_{z}=\sum_{m=-M}^{M} \xi_{m}^{0}(z) g_{m}
$$

where the functions $g_{m}$ are independent from $z$ and are solutions to:

$$
\left(F^{*} F+\alpha_{z} I\right) g_{m}=F^{*} \psi_{m}
$$

Practical experiments suggest that generally $M$ does not need to be large (due to the behavior of Bessel functions) and, in particular the needed $M$ is much smaller than the dimension of the grid points for $z$. 


\section{References}

[1] Riccardo Aramini, Massimo Brignone, Michele Piana, The linear sampling method without sampling, Inverse Problems 22 2237-2254, 2006.

[2] Fioralba Cakoni, David Colton, Qualitatives methods in inverse scattering theory, Springer, 2006.

[3] Fioralba Cakoni, David Colton, Peter Monk On the use of transmission eigenvalues to estimate the index of refraction from farfield data, Inverse Problems pp. 507 - 522, 2007.

[4] Fioralba Cakoni, David Colton, Houssem Haddar The computation of lower bounds for the norm of the index of refraction in an anisotropic media from farfield data, J. Integral Equations and Applications, 2008.

[5] Fioralba Cakoni, David Colton, Houssem Haddar On the determination of Dirichlet or transmission eigenvalues from farfield data, Comptes Rendus Mathematique, 348, no 7-8, 379-383, (2010).

[6] Fioralba Cakoni, Drossos Gintides, Houssem Haddar The existence of an infinite descrete set of transmission eigenvalues, SIAM J. Math. Analysis, 42, no 1, 237-255 (2010).

[7] David Colton, Houssem Haddar, Michele Piana The linear sampling method in inverse electromagnetic scattering theory, Inverse Problems, 2003.

[8] David Colton, Rainer Kress, Inverse acoustic and electromagnetic scattering theory, second edition, Berlin: Springer, 1998.

[9] David Colton, Peter Monk, A linear sampling method for the detection of leukemia using microwaves, Siam J. Appl. Math. pp. 926-941, 1998.

[10] David Colton, Lassi Päivärinta, John Sylvester The interior transmission problem, Inverse Problems and Imaging Vol. 1, 13 - 28, 2007.

[11] David Colton, Peter Monk, and Jiguang Sun. Analytical and computational methods for transmission eigenvalues. Inverse Problems, 26(4):045011, 16, 2010.

[12] David Colton, Michele Piana The simple method for solving the electromagnetic inverse scattering problem: the case of TE polarized waves, Inverse Problems 14, 597-614, 1998.

[13] David Colton, Michele Piana, Roland Potthast, A simple method using Morozov's discrepancy principle for solving inverse scattering problems, Inverse Problems 13, 1477-1493, 1997.

[14] Andreas Kirsch and Natalia Grinberg. The factorization method for inverse problems, volume 36 of Oxford Lecture Series in Mathematics and its Applications. Oxford University Press, Oxford, 2008.

[15] Lassi Päivärinta, John Sylvester Transmission eigenvalues, SIAM journal on Mathematical Analysis Vol. 40 (2), 2008.

[16] FreeFem++ (http://www.freefem.org/ff++/) 


\section{Contents}

\begin{tabular}{|lr}
\hline 1 & Introduction \\
\hline
\end{tabular}

\begin{tabular}{|ll|}
\hline 2 & Forward and inverse scattering problem \\
\hline
\end{tabular}

3 Derivation of a constant approximation of the refractive index 6

3.1 Transmission eigenvalues and their identification using farfield data . . . . . . . . . 6

3.2 Identification of the refractive index $\ldots \ldots \ldots \ldots$. . . . . . . . . . 7

3.3 The case of an inhomogeneous background . . . . . . . . . . . . . . . . . . . 8

3.3.1 A remark on the case of absorbing background . . . . . . . . . . . . . . . . 9

4 The case of TE polarization 10

4.1 Transmission eigenvalues . . . . . . . . . . . . . . . . . . . . . . . 10

4.2 Mixed formulations of the refractive index eigenvalues problems . . . . . . . . . . . 10

4.2.1 The case of an inhomogeneous background . . . . . . . . . . . . . 11

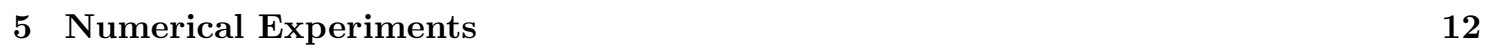

5.1 The case where the shape is known . . . . . . . . . . . . . . . . . . . 12

5.2 Numerical results for cases where the geometry is not known a priori . . . . . . . . 17

5.2 .1 The case of circular geometries. . . . . . . . . . . . . . . . 17

5.2 .2 Cases of non circular geometries . . . . . . . . . . . . . . . . . . . . . . . . . . . .

5.2 .3 Cases of multiply connected inclusions . . . . . . . . . . . . . . . . 22

A Fast implementation of LSM in the case of a background with axisymmetric index

References 27 
Centre de recherche INRIA Saclay - Île-de-France

Parc Orsay Université - ZAC des Vignes

4, rue Jacques Monod - 91893 Orsay Cedex (France)

Centre de recherche INRIA Bordeaux - Sud Ouest : Domaine Universitaire - 351, cours de la Libération - 33405 Talence Cedex Centre de recherche INRIA Grenoble - Rhône-Alpes : 655, avenue de l'Europe - 38334 Montbonnot Saint-Ismier

Centre de recherche INRIA Lille - Nord Europe : Parc Scientifique de la Haute Borne - 40, avenue Halley - 59650 Villeneuve d'Ascq Centre de recherche INRIA Nancy - Grand Est : LORIA, Technopôle de Nancy-Brabois - Campus scientifique 615, rue du Jardin Botanique - BP 101 - 54602 Villers-lès-Nancy Cedex

Centre de recherche INRIA Paris - Rocquencourt : Domaine de Voluceau - Rocquencourt - BP 105 - 78153 Le Chesnay Cedex

Centre de recherche INRIA Rennes - Bretagne Atlantique : IRISA, Campus universitaire de Beaulieu - 35042 Rennes Cedex

Centre de recherche INRIA Sophia Antipolis - Méditerranée : 2004, route des Lucioles - BP 93 - 06902 Sophia Antipolis Cedex 